\title{
Why are periodic erythrocytic diseases so rare in humans?
}

\begin{abstract}
Many studies have shown that periodic erythrocytic (red blood cell linked) diseases are extremely rare in humans. To explain this observation, we develop here a simple model of erythropoiesis in mammals and investigate its stability in the parameter space. A bifurcation analysis enables us to sketch stability diagrams in the plane of key parameters. Contrary to some other mammal species such as rabbits, mice or dogs, we show that human specific parameter values prevent periodic oscillations of red blood cells levels. In other words, human erythropoiesis seems to lie in a region of parameter space where oscillations exclusively concerning red blood cells cannot appear. Further mathematical analysis show that periodic oscillations of red blood cells levels are highly unusual and if exist, might only be due to an abnormally high erythrocytes destruction rate or to an abnormal hematopoietic stem cell commitment into the erythrocytic lineage. We also propose numerical results only for an improved version of our approach in order to give a more realistic but more complex approach of our problem.
\end{abstract}

\author{
ADIMY Mostafa \\ mostafa.adimy@inria.fr / tél. (+33)(0) 4.72.43.74.88 \\ INRIA Antenne Lyon la Doua, Bâtiment CEI-2, 56, Boulevard Niels Bohr, CS 52132, \\ F-69622 Villeurbanne cedex, France \\ http://math.univ-lyon1.fr/ adimy/
}

BABIN Louis

louis.babin@etu.univ-lyon1.fr

Université de Lyon, Université Claude Bernard Lyon 1

PUJO-MENJOUET Laurent

pujo@math.univ-lyon1.fr / tél. (+33)(0) 4.72.43.10.08

Université de Lyon, Université Claude Bernard Lyon 1, CNRS UMR 5208, Institut Camille Jordan, 43 blvd. du 11 novembre 1918, F-69622 Villeurbanne cedex, France

http://math.univ-lyon1.fr/ pujo/

Keywords Erythropoiesis, Periodic hematological diseases, Dynamic Diseases, Delay Differential Equations, Hopf Bifurcation

Mathematical Subject Classification 34K18, 34K20, 37G15, 37N25, 92C30, 92D25

\section{Introduction}

A medullar enigma : why are cyclic diseases exclusively concerning human erythrocytes so rare?

Erythropoiesis, along with leukopoiesis and thrombopoiesis, are subparts of hematopoiesis which accounts for the medullar production and regulation of the three blood cell types : red blood cells or erythrocytes, white blood cells or leukocytes and platelets or thrombocytes.

In some hematopoietic disorders labeled as dynamical hematological diseases by Mackey and Glass (1977), blood cell production operates in a region of physiological parameters that leads to pathological behavior and abnormalities in blood cell counts. Such physiological behavior would originate either 
from bone marrow defects or from peripheral origins (Haurie et al., 1998). Disregarding the causes, periodic oscillations in blood cell levels can appear and result in a so called cyclic hematological disorder. The occurrence of such periodic fluctuations specific to hematopoietic stem cells (HSC), leukocytes and thrombocytes has been clearly established and documented (for reviews see e.g. Reimann, 1971; Haurie et al., 1998; Foley and Mackey, 2009). Famous examples of periodic hematological disorders are cyclical neutropenia (see e.g. Dale and Hammond, 1988), periodic chronic myelogenous leukemia (see e.g. Morley et al., 1967) and cyclic thrombocytopenia (see e.g. Fontenele et al., 2015). However, when regarding erythropoiesis, rare oscillations of some erythrocytic cell types have effectively been reported, but the situation seems different. Actually, these oscillations are often coupled either with oscillations from other blood cell lineages (see e.g. Morley, 1969, 1979) or with cyclic syndromes such as periodic auto immune hemolytic anemia (Ranløv and Videbæk, 1963; Meyer et al., 1978; Björkholm et al., 1982). These situations indicate that periodic erythrocytic oscillations originate either from the hematopoietic stem cell population, impacting then all the lineages (see e.g. Haurie et al., 1998) or from the periodic occurrence of a syndrome. Interestingly, specific only erythropoiesis cyclic fluctuations have rarely been documented. It has only been reported for few cases in animals : clinically observed in dogs (Morley and Stohlman, 1969) and experimentally induced in rabbits and mice (Orr et al., 1968; Gurney et al., 1981; Gibson et al., 1984, 1985). Even rarer in humans, it has only been reported in a young patient with pure red cell aplasia (Gordon and Varadi, 1962) with very long periods (between three to five months). One can thus be surprised by such exceptional and unusual observations.

In this work, we attempt then to explain this through a simple model of erythropoiesis.

\section{How to choose a simple but relevant model?}

Many models of hematopoiesis already exist (see e.g. Pujo-Menjouet, 2016) some may deal with the production and regulation of one single hematopoietic lineage (see e.g. Craig et al., 2016; Langlois et al., 2017) while others focus on the hematopoietic stem cell population (see e.g. Mackey, 1978, 2001; Pujo-Menjouet et al., 2005; Adimy et al., 2005, 2006). Blood cell production is believed to involves delays due to maturation, proliferation and feedback processes and requires thus the use of delay differential equations (DDE) (see e.g. Mackey and Glass, 1977; Foley and Mackey, 2009). Some models, although carefully designed use DDEs without mathematically deriving the appearance of delays (see e.g. Loeffler et al., 1989; Ramakrishnan et al., 2004; Woo et al., 2006; Krzyzanski et al., 2008), while age-structured models, such as Fuertinger et al. (2013), composed of partial differential equations (PDEs) of transport type allow clear mathematical derivation of DDEs thanks to an integration with respect to the structure variables (see e.g. Banks et al., 2004) and the use of the methods of characteristics. Many models have already been developed to account for some periodic hematological disorders (for a detailed review see Foley and Mackey, 2009). In this present work, we focus on cyclic erythropoiesis and design a model to explain this rarely observed disorder. To that purpose, we take advantage of relevant erythropoiesis mice, dogs and rabbit models (Bélair et al., 1995; Mahaffy et al., 1998; Fuertinger et al., 2013) and mathematical analysis specific to DDEs (Beretta and Kuang, 2002; Adimy et al., 2005; Crauste, 2010; Boullu et al., 2019a) Changes on parameter values accounting for the cyclic erythropoiesis have been well described: among these changes, one can note an increase of the hematopoietic stem cells apoptosis rate (Mackey, 1997) (mice and dogs) or a death rate amplification of mature red blood cells in the case of induced auto immune hemolytic anemia (Mackey, 1979) (rabbits). However, erythrocyte periodic oscillations specific to humans have been poorly investigated. Our approach is based on the work of Mackey (1979). In Section 2, we set up an updated erythropoiesis model and, in Section 3, prove its well-posedness. Section 4 is dedicated to the analysis of the asymptotic behavior with respect to the variation of four parameters. In Section 5, we apply our model to the occurrence of periodic oscillations for rabbits and humans. Then, in Section 6, we detail further improvement and suggestions. We conclude this paper with a discussion in Section 7. 


\section{$2 \quad$ Erythropoiesis Modeling}

\subsection{Red blood cell production, destruction and regulation}

Before designing our model, let us briefly remind the biological background. The process begins with a pool of pluripotent myeloid hematopoietic stem cell able to self-renew or differentiate into three different hematopoietic lineages (erythrocytic, megakaryocytic and granulocytic). The irreversible commitment into the erythrocytic lineage leads to committed erythroid progenitor cells called Burst Forming Unit Erythroid (BFU-E) and the rapidly dividing Colony Forming Unit Erythroid (CFU-E). Under normal conditions in humans, cells stay in these two stages for about 13 to 14 days while they differentiate and divide (about five to seven times). Further differentiation leads the second identifiable compartment of erythroid precursors called normoblasts or erythroblasts with four to five more divisions. It takes approximately five days (Beutler et al., 2001; Doig, 2015) for these cells to lose their nucleus and become marrow reticulocytes and leave the bone marrow - in 1 to 2 days - to reach the peripheral blood. During the transit in the bone marrow - from the proerythroblast to reticulocyte stages - hemoglobin is synthesized.

However red blood cells are continuously destroyed due to intra or extra vascular processes occurring both in normal and pathological situations.

To maintain essential life functions, these losses have to be permanently compensated for. Erythrocytes production must be carefully regulated. This regulation is mainly performed by erythropoietin (EPO) : an hormone essentially produced in kidneys. In normal physiological conditions, thanks to $\mathrm{O}_{2}$ sensors in kidneys, EPO production is negatively regulated by the $\mathrm{O}_{2}$ level of blood. Consequently, the total number of erythrocytes is in negative feedback with EPO concentrations. Erythropoiesis is thus a complex controlled system which should ensure that the red blood cell count and hemoglobin level stay within narrow limits. For instance, in humans, hemoglobin concentration should steadily remain, without abrupt changes, within 11.5 and 13.5 g.dL $\mathrm{dL}^{-1}$ which corresponds roughly to an interval of $3 \times 10^{11}$ to $4.5 \times 10^{11}$ red blood cells for each kilogram of body weight. Abnormally low (anemia), high (absolute polycythemia) or oscillating levels of hemoglobin are harmful and health could be greatly impaired.

\subsection{Erythropoiesis Model}

Our new model is based on biological updates illustrated in Fig. 1 with parameters specified in Table 1.

\subsubsection{Bone marrow cell compartment}

Our erythropoiesis model begins with the compartment of the maturing and differentiating bone marrow cells. Cells transit in this compartment for a time $\tau \geq 0$ while some of them die randomly with a rate $\delta_{m e}>0$. This rate accounts both for the apoptosis of erythroid progenitors (essentially of $C F U-E$ ) and for the ineffective erythropoiesis possibly due to iron deficiency (Fuertinger et al., 2013) which occurs even in normal physiological conditions (Finch et al., 1970; Dinant and Maat, 1978; Beutler et al., 2001; Lichtman et al., 2006).

Even though EPO normally downregulates erythropoiesis by preventing erythroid progenitor from apoptosis (see e.g. Koury and Bondurant, 1990; Wu et al., 1995; Doig, 2015) $\delta_{m e}$ is assumed to be constant for simplification purpose. Insight in the effect of EPO could be seen by a variation of this parameter investigated in Section 4.2.

Let $m(t, a)$ be the population density of bone marrow cells at time $t \geq 0$ with the age $a \in(0, \tau)$. The age-structured equation ruling the density $m$ is

$$
\frac{\partial m}{\partial t}(t, a)+\frac{\partial m}{\partial a}(t, a)=-\delta_{m e} m(t, a), t \geq 0, a \in(0, \tau),
$$




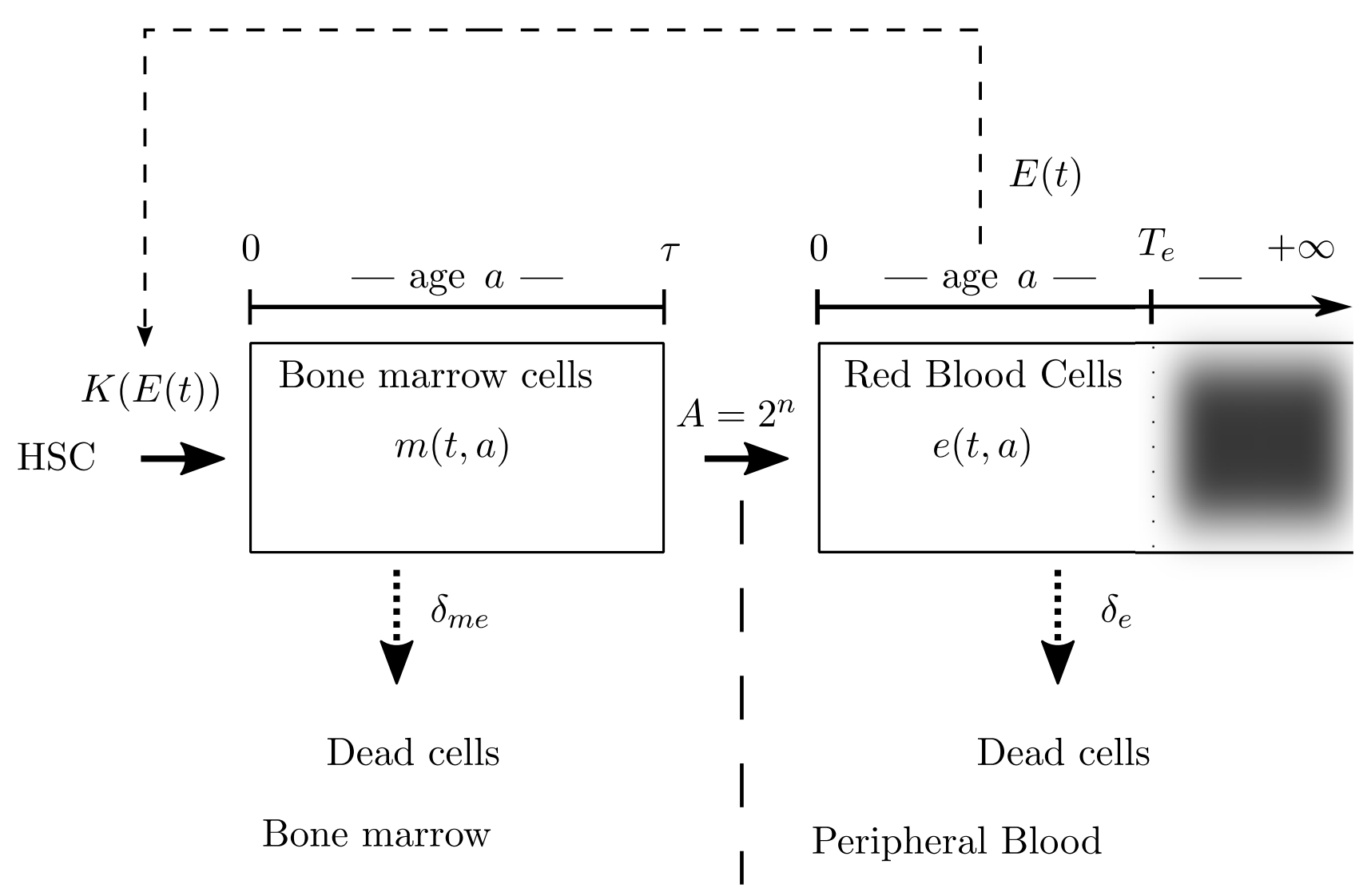

Figure 1 Erythropoiesis model organized in two aged-structured compartments describing populations of Bone marrow cells and Red Blood Cells (RBCs). When a cell enters one compartment, its age is reset to 0 . This explains why age is a structured variable. The maximal age a cell spends in the bone marrow compartment is $\tau$ and it is assumed to be $+\infty$ for RBCs (even if it is 120 days, we assume that this is large enough and very few cells survive after that time, represented by the shaded dark zone). Population death rates are $\delta_{m e}$ and $\delta_{e}$. The amplification parameter $A=2^{n}$ translates the $n \in \mathbb{N}$ mitotic divisions undergone by a single nucleated cell during its development in the bone marrow. $T_{e}$ represents the mean lifespan of red blood cells and essentially accounts for extravascular senescence. The kinetics of EPO does not appear explicitly here. Indeed, we consider that the red blood cell population directly downregulates erythropoiesis. We decide to model this downregulation through a negative feedback of the total red blood cell population - $E$ - over the cell influx $K(E)$ coming from the Hematopoietic Stem Cell population. 
Table 1 Signification and possible values of model parameters. Possible values are either exact or approximated values from literature. References are specified as numbers for concision: 1: Jandl (1987), 2: Beutler et al. (2001), 3: Beutler et al. (2001), 4: Lichtman et al. (2006), 5: Lodish et al. (2010), 6: Fuertinger et al. (2013), 7: Doig (2015), 8: Rogg et al. (2019), 9: Butina (2020), 10: Erslev (1997), 11: Mackey (1979), 12: Crauste et al. (2008), 13: Myssina et al. (2003), 14: Dou et al. (2012), 15: Sato et al. (2012), 16: Ma et al. (2017), 17: Bélair et al. (1995), 18: Greer et al. (2003), 19: Mackey (2001), 20: Finch (1982), 21: Goodnough (2002)

\begin{tabular}{|c|c|c|c|c|}
\hline Name & Signification & Possible values & Units & References \\
\hline$\tau$ & $\begin{array}{l}\text { Bone marrow tran- } \\
\text { sit time }\end{array}$ & {$[14,22]$} & days & $\begin{array}{l}1,2,3,4,5 \\
6,7,8,9\end{array}$ \\
\hline$\delta_{m e}$ & $\begin{array}{l}\text { Death rate of bone } \\
\text { marrow cells }\end{array}$ & {$[0,1,1]$} & day $^{-1}$ & $3,4,6,8,10$ \\
\hline$\delta_{e}$ & Death rate of RBCs & {$[0,01,0,04]$} & day $^{-1}$ & $4,6,11,12$ \\
\hline$T_{e}$ & $\begin{array}{l}\text { Mean lifespan of } \\
\text { RBCs }\end{array}$ & {$[60,120]$} & days & $\begin{array}{l}1,4,6,13 \\
14,15,16\end{array}$ \\
\hline$\delta_{e, b}$ & $\begin{array}{l}\text { Auxiliary death } \\
\text { rate }\end{array}$ & {$[0,001,0,01]$} & day $^{-1}$ & $2,6,7,17$ \\
\hline $\begin{array}{ll}n & \& \\
A & = \\
2^{n} & \end{array}$ & $\begin{array}{l}\text { Number of mitotic } \\
\text { divisions \& amplifi- } \\
\text { cation parameter }\end{array}$ & {$[5,11]$} & - & $2,6,7,9,18$ \\
\hline$K_{c}$ & $\begin{array}{l}\text { Basal influx of } \\
\text { HSCs }\end{array}$ & {$\left[10^{7}, 5.10^{9}\right]$} & cells. $\mathrm{kg}^{-1} \cdot$ day $^{-1}$ & $\begin{array}{l}\text { Estimated } \\
\text { from } 6,11, \\
12,17,19\end{array}$ \\
\hline$\alpha$ & $\begin{array}{l}\text { Supplementary in- } \\
\text { flux of HSCs }\end{array}$ & {$\left[3 K_{c}, 6 K_{c}\right]$} & cells. $\mathrm{kg}^{-1} \cdot$ day $^{-1}$ & $\begin{array}{l}\text { Estimated } 6, \\
12,17,20,21\end{array}$ \\
\hline$\beta$ & $\begin{array}{l}\text { RBC quantity to } \\
\text { reach HSCs median } \\
\text { influx. }\end{array}$ & {$\left[10^{11}, 10^{12}\right]$} & cells. $\mathrm{kg}^{-1}$ & $\begin{array}{l}\text { Estimated } \\
\text { from } 6,12, \\
17\end{array}$ \\
\hline$q$ & $\begin{array}{l}\text { Hill function "sensi- } \\
\text { tivity" parameter }\end{array}$ & - & - & $\begin{array}{l}\text { Arbitrary } \\
\text { and inspired } \\
\text { from } 11,12, \\
17\end{array}$ \\
\hline
\end{tabular}


and the total population of bone marrow cells is given by

$$
M(t)=\int_{0}^{\tau} m(t, a) \mathrm{d} a .
$$

This compartment is supplied with a cell flux $K$ coming from a pool of hematopoietic stem cells whose population dynamic is not modeled here. Recent works of Grover et al. (2014) and Singh et al. (2018) seem to indicate that EPO stimulates the commitment of hematopoietic stem cells into the erythrocytic lineage. Even though this idea remains to be investigated, we decide, for simplicity, to only take this feedback process into account. Moreover, as mentioned in Section 2.1, EPO is negatively regulated by the total number of red blood cells. Indeed, to simplify, we assume that feedback reactions are fast compared to other physiological mechanism. It enables us not to model the kinetic of EPO. All things considered, we model the supply of bone marrow cells $K(\cdot)$ as a decreasing function of the total population of red blood cells - E - such that :

$$
K(E)=K_{c}+\frac{\alpha}{1+\left(\frac{E}{\beta}\right)^{q}},
$$

where $K_{c} \geq 0$ represents the basal cell flux of hematopoietic stem cells when the total number of red blood cells is large $(E \rightarrow+\infty)$. Parameter $\alpha>0$ stands for the supplementary influx due to a decrease in red blood cell population $(E \rightarrow 0)$, while $\beta>0$ is the total number of red blood cells corresponding to the median flux $K_{c}+\frac{\alpha}{2}$ and $q \geq 1$ is the Hill coefficient characterizing how fast is the change between erythrocytes number and hematopoietic stem cells commitment. It is difficult to find the value of these parameters in the literature. Thus, as in numerous modeling works (see e.g. Mackey, 1979; Fuertinger et al., 2013), we set them accordingly to the fact that erythropoiesis is expected to increase about three to fivefold (Finch, 1982; Goodnough, 2002). Consequently, we assume that $\alpha \in\left[3 K_{c}, 6 K_{c}\right]$. The boundary condition of this compartment is then given by

$$
m(t, 0)=K(E(t)), t \geq 0 .
$$

Additionally, while going through this compartment, a single cell undergoes $n$ mitotic divisions. In our model, we take this amplification into account at the end the bone marrow cells compartment where we assume that the cell density $m(t, \tau)$ is amplified by a factor $A=2^{n}$ before entering the blood circulatory compartment.

\subsubsection{Red Blood Cell compartment}

The model ends with the compartment of the red blood cells which describes the population of cells who lie in the peripheral blood that is peripheral blood reticulocytes and erythrocytes.

Red blood cell degradation is crucial in erythropoiesis and involves different complex processes. It is thus important to model erythrocyte destruction carefully. To do so, we analyzed numerous different erythropoiesis models (see e.g. Bélair et al., 1995; Mahaffy et al., 1998; Fuertinger et al., 2013). We decided, for the sake of simplicity, to model erythrocytes removal by a non specific death rate $\delta_{e}>0$ that accounts for every cause of death. Doing so, selective removal of senescent red blood cells is not modeled in detail but we still try to keep a relevant biological model by expressing $\delta_{e}$ such that :

$$
\delta_{e}=\frac{\ln 3}{T_{e}}+\delta_{e, b}
$$

where $T_{e}$ is the mean lifespan of erythrocytes and $\frac{\ln 3}{T_{e}}$ accounts for senescence. Thus $\ln (3) / T_{e}$ ensures that at least two thirds of the RBCs that entered the compartment at time $t$ have been destroyed a time $T_{e}>0$ thereafter. The term $\delta_{e, b}$ is an auxiliary death rate, usually smaller than $\frac{\ln 3}{T_{e}}$, that accounts for cell destruction processes different from senescence (due to infrequent intravascular hemolysis or blood losses). With this assumption, the age of cells can possibly go until infinity even though a large majority has been destroyed at age $T_{e}$. 
All things considered, if $e(t, a)$ is the population red cells density at time $t \geq 0$ with age $a>0$, the age-structured equation of transport ruling its dynamics is given by

$$
\frac{\partial e}{\partial t}(t, a)+\frac{\partial e}{\partial a}(t, a)=-\delta_{e} e(t, a), t \geq 0, a>0,
$$

and the total population is given by

$$
E(t)=\int_{0}^{+\infty} e(t, a) \mathrm{d} a,
$$

with the boundary condition

$$
e(t, 0)=A m(t, \tau) .
$$

Using the framework of Eq. (5) and the classical methods of characteristics, we obtain that at long times, if $t>T_{e}$, the number of cells entering the compartment at time $t-T_{e}$ and who survived $T_{e}$ days later is such that

$$
e\left(t, T_{e}\right)=e\left(t-T_{e}, 0\right) \mathrm{e}^{-\delta_{e} T_{e}} .
$$

From this expression and Eq. (4), we see that the term $\frac{\ln 3}{T_{e}}$ effectively models senescence. It ensures that at least two thirds of the red blood cells that entered the compartment at time $t$ have been destroyed a time $T_{e}>0$ thereafter:

$$
e\left(t, T_{e}\right) \leq \frac{2}{3} e\left(t-T_{e}, 0\right)
$$

\section{Reduction of the model}

Assuming that the solutions are smooth enough, we integrate the equations (1) and (5) over the age variable and we take into account their associated boundary conditions (3) and (6). It simply leads to the following system of differential equations if $t \geq 0$ :

$$
\begin{aligned}
M^{\prime}(t) & =-m(t, \tau)+K(E(t))-\delta_{m e} M(t), \\
E^{\prime}(t) & =-\delta_{e} E(t)+A m(t, \tau),
\end{aligned}
$$

whose short time dynamics (not mentioned here) is easily solved with the knowledge of initial population densities and initial conditions given by

$$
M(0)=M_{0}=\int_{0}^{\tau} m(0, a) \mathrm{d} a, \quad E(0)=E_{0}=\int_{0}^{+\infty} e(0, a) \mathrm{d} a .
$$

We simply focus on the long time dynamics, for $t \geq \tau$. Thanks to the classical method of characteristics and the boundary condition (3), we obtain

$$
m(t, \tau)=m(t-\tau, 0) \mathrm{e}^{-\delta_{m e} \tau}=K(E(t-\tau)) \mathrm{e}^{-\delta_{m e} \tau}
$$

from which we deduce

$$
M^{\prime}(t)=-K(E(t-\tau)) \mathrm{e}^{-\delta_{m e} \tau}+K(E(t))-\delta_{m e} M(t), \text { if } t \geq \tau .
$$

Hence, the dynamics of the red blood cell population $E$ entirely rules the dynamics of the bone marrow cell population $M$ as follows

$$
M(t)=\int_{0}^{\tau} K(E(t-u)) \mathrm{e}^{-\delta_{m e} u} \mathrm{~d} u .
$$

All in all, the time behavior of the model relies on the equation ruling the erythrocyte population dynamics

$$
E^{\prime}(t)=-\delta_{e} E(t)+A K(E(t-\tau)) \mathrm{e}^{-\tau \delta_{m e}} .
$$


We want to study its steady states. They verify

$$
\delta_{e} E=A \mathrm{e}^{-\tau \delta_{m e}} K(E), E \geq 0 .
$$

Due to the variation of $K$, Eq. (8) has a unique solution and Eq. (7) consequently admits a unique equilibrium $E^{*}>0$. These elements and the implicit function theorem enable us to state the following Proposition.

Proposition 3.1. Eq. (7) admits a unique positive equilibrium $E^{*}$ which satisfies

$$
\delta_{e} E^{*}=A \mathrm{e}^{-\tau \delta_{m e}} K\left(E^{*}\right)=A \mathrm{e}^{-\tau \delta_{m e}}\left(K_{c}+\frac{\alpha}{1+\left(\frac{E^{*}}{\beta}\right)^{q}}\right),
$$

and consequently verifies

$$
\frac{A \mathrm{e}^{-\tau \delta_{m e}} K_{c}}{\delta_{e}} \leq E^{*} \leq \frac{A \mathrm{e}^{-\tau \delta_{m e}}\left(K_{c}+\alpha\right)}{\delta_{e}} .
$$

Moreover, $E^{*}$ is a continuously differentiable function with respect to all parameters. It is especially decreasing with respect to $\delta_{e}, \delta_{m e}$ and $\tau$ and increasing with respect to $K_{c}$.

The associated bone marrow population $M^{*}>0$ is also unique and verifies

$$
M^{*}=\frac{K\left(E^{*}\right)\left[1-\mathrm{e}^{-\tau \delta_{m e}}\right]}{\delta_{m e}} .
$$

The derivatives of $E^{*}$ with respect to $\tau, \delta_{e}, K_{c}$ and $\delta_{m e}$ are detailed in Appendix 2.

Remark 1. Solving Eq. (1) and Eq. (5) along with the stationary condition and

$$
E^{*}=\int_{0}^{+\infty} e^{*}(a) \mathrm{d} a
$$

gives the stationary population densities $-m^{*}$ and $e^{*}$ - associated to the equilibrium $\left(M^{*}, E^{*}\right)$ :

$$
\begin{aligned}
& m^{*}(a)=K\left(E^{*}\right) \mathrm{e}^{-\delta_{m e} a}, \quad a \in[0, \tau], \\
& e^{*}(a)=A \mathrm{e}^{-\tau \delta_{m e}} K\left(E^{*}\right) \mathrm{e}^{-\delta_{e} a}=\delta_{e} E^{*} \mathrm{e}^{-\delta_{e} a}, \quad a \in[0,+\infty) .
\end{aligned}
$$

\section{Local asymptotic stability of the steady state}

Let us now perform a local asymptotic stability analysis of Eq. (7) near its unique equilibrium $E^{*}$ and obtain a classical characteristic equation (Section 4.1). Analyzing the roots of this equation enables us to investigate the stability of $E^{*}$ in the space of parameters (Section 4.2). We especially focus on $\tau, \delta_{e}, K_{c}$ and $\delta_{m e}$ and show that - under some conditions - periodic solutions may appear and potentially through a Hopf bifurcation. Stability diagrams in the space of parameters as well as example trajectories were drawn using MATLAB and the function dde23 (Shampine and Thompson, 2001).

\subsection{Linearization about the equilibrium}

After a simple translation of the time variable, Eq. (7) is linearized about its unique steady state $E^{*}>0$ and gives

$$
z^{\prime}(t)=-\delta_{e} z(t)-L z(t-\tau), t \geq 0
$$

where

$$
\begin{aligned}
L & =-A \mathrm{e}^{-\tau \delta_{m e}} \frac{\partial K}{\partial E}\left(E^{*}\right), \\
& =q \frac{A \mathrm{e}^{-\tau \delta_{m e}}}{\beta}\left(\frac{E^{*}}{\beta}\right)^{q-1} \frac{\alpha}{\left(1+\left(\frac{E^{*}}{\beta}\right)^{q}\right)^{2}},
\end{aligned}
$$


is strictly positive and continuously differentiable with respect to all the parameters. Its corresponding derivatives with respect to $\tau, \delta_{e}, K_{c}$ and $\delta_{m e}$ are detailed in Appendix 2.

Every solution of Eq. (11) can be decomposed as an infinite linear combination of eigenfunctions of the form $z_{\lambda}: t \mapsto \exp (\lambda t)$ associated to the eigenvalue $\lambda \in \mathbb{C}$. Introducing the expression of $z_{\lambda}$ into Eq. (11), leads to the characteristic equation of Eq. (7) linearized about $E^{*}$ :

$$
\lambda+\delta_{e}+L e^{-\lambda \tau}=0, \lambda \in \mathbb{C} .
$$

This equation being transcendental, determining the position in the complex plane of its roots $\lambda \in \mathbb{C}$ is tricky. To study it, we begin with the formulation of a sufficient stability condition for the equilibrium $E^{*}$.

Proposition 4.1 (Hayes (1950)). If $\tau=0$ or $L \leq \delta_{e}$ then all roots of Eq. (12) have negative real parts.

The proof of this proposition is mentioned in Appendix 1. Keeping in mind that $L$ is an implicit function of model parameters, the latter condition enables us to identify regions in parameter space where $E^{*}$ is locally asymptotically stable.

Actually, as long as model parameters vary while satisfying Proposition $4.1, E^{*}$ is stable and stability switches cannot occur. However, when Proposition 4.1 does not hold anymore, solution dynamics is unknown.

In the following, we investigate the local asymptotic stability of $E^{*}$ when model parameters do not verify Proposition 4.1. We wish to identify the boundaries in parameter space where stability switches could occur due to a variation of one of the model parameters -such as $\tau, \delta_{e}, \delta_{m e}$ or $K_{c^{-}}$all the others being fixed by using techniques of Beretta and Kuang (2002); Adimy et al. (2005, 2006); Adimy and Crauste $(2007,2012)$ and Boullu et al. (2019a,b).

\subsection{Local stability with respect to parameter variations}

Denote by $\psi \in \mathcal{P}$ one of the parameters among $\delta_{e}, K_{c}, \delta_{m e}$, or $\tau$. $\mathcal{P}$ is the set of possible values for the chosen parameter $\psi$ (the only one to be varied, other parameters being fixed to given values). For the sake of simplicity, in this section we only explicit the dependence with respect to the parameter $\psi$. First, we define the set

$$
\Pi=\left\{\psi \in \mathcal{P} \mid 0<\delta_{e}<L(\psi)\right\},
$$

gathering all possible values of the parameter $\psi$ for which Proposition 4.1 is not verified (assuming that $\tau>0)$. We subsequently simply obtain:

Corollary 4.1. If $\psi \notin \Pi$ then all roots $\lambda \in \mathbb{C}$ of $E q$. (12) have negative real parts and $E^{*}(\psi)$ is thus locally asymptotically stable.

All other parameters being fixed, the set $\Pi$ consequently defines the set of parameter values in which stability switches could occur. From the variations of $L$ with respect to $\psi$, we identify sufficient conditions ensuring the existence of $\Pi$ and enabling to give it a more explicit form.

In this work, we studied the effect of $\delta_{e}, K_{c}, \delta_{m e}$ and $\tau$ on the local asymptotic stability of $E^{*}$. We thus established the variations of $L$ with respect to each of these parameters thanks to its derivatives. We obtained the following properties:

(i) For $\psi=\delta_{e}$, we note $\Pi=J$ and obtain:

Proposition 4.2. $J \neq \emptyset$ if and only if

$$
\frac{\alpha}{K_{c}}>\frac{4 q}{(q-1)^{2}} .
$$

In this case,

$$
J=\left(\delta_{e, \min }, \delta_{e, \max }\right)
$$

where $\delta_{e, \min }<\delta_{e, \max }$ are uniquely defined such that $L\left(\delta_{e, \max }\right)=L\left(\delta_{e, \min }\right)=\delta_{e}$ and $L\left(\delta_{e}\right) \geq \delta_{e}$ for all $\delta_{e} \in\left[\delta_{e, \min }, \delta_{e, \max }\right]$. 
(ii) For $\psi=K_{c}$, we note $\Pi=\mathcal{K}$ and obtain:

Proposition 4.3. If

$$
\frac{4 q}{q^{2}-1}\left(\frac{q-1}{q+1}\right)^{\frac{1}{q}}<\frac{A \alpha \mathrm{e}^{-\tau \delta_{m e}}}{\beta \delta_{e}} \leq\left(\frac{q-1}{q+1}\right)^{\frac{1}{q}},
$$

then $\mathcal{K} \neq \emptyset$ et $\mathcal{K} \subset\left[0, K_{c, \max }\right)$ where $K_{c, \max } \in\left[\tilde{K}_{c},+\infty\right)$ is defined such that $L\left(K_{c, \max }\right)=\delta_{e}$ and $L\left(K_{c}\right)>\delta_{e}$ for all $K_{c} \in \mathcal{K} \cap\left[0, K_{c, \max }\right)$. Furthermore, if $L\left(K_{c}=0\right) \leq \delta_{e}$ then we uniquely define $K_{c, \min }$ and $K_{c, \max }$ such that $K_{c, \min }<K_{c, \max }$ and

$$
\mathcal{K}=\left(K_{c, \min }, K_{c, \max }\right) .
$$

(iii) For $\psi=\delta_{m e}$, we note $\Pi=\mathcal{D}$ and obtain:

Proposition 4.4. If

$$
\frac{A K_{c}}{\delta_{e}}>\beta
$$

and

$$
\frac{(q-1) \alpha}{2 K_{c}}>1+\sqrt{1+\frac{\alpha}{K_{c}}},
$$

then

$$
D=\left(\delta_{m e, \min }, \delta_{\text {me,max }}\right),
$$

where $\delta_{m e, \text { min }}<\delta_{\text {me,max }}$ are uniquely defined such that $L\left(\delta_{m e, \max }\right)=L\left(\delta_{m e, \min }\right)=\delta_{e}$ and $L\left(\delta_{m e}\right) \geq$ $\delta_{e}$ for all $\delta_{m e} \in\left[\delta_{m e, m i n}, \delta_{m e, m a x}\right]$.

(iv) For $\psi=\tau$, we note $\Pi=I$ and obtain:

Proposition 4.5. If the conditions (14) and (15) hold then $I \neq \emptyset$ and there exists a unique $\tau_{\max } \in$ $[\tilde{\tau},+\infty)$ such that $L\left(\tau_{\max }\right)=\delta_{e}$ and $L(\tau)>\delta_{e}$ for all $\tau \in I \cap\left[0, \tau_{\max }\right)$, such that $I \subset\left[0, \tau_{\max }\right)$. Furthermore, if $L(\tau=0) \leq \delta_{e}$ then we uniquely define $\tau_{\min } \in[0, \tilde{\tau}]$ such that $L\left(\tau_{\min }\right)=\delta_{e}$ and

$$
I=\left(\tau_{\min }, \tau_{\max }\right) .
$$

Thus, we identified sufficient conditions ensuring that $\Pi \neq \emptyset$ and that $\mathcal{P} \backslash \Pi \neq \emptyset$. In other words, if these conditions hold, then there exist some values $\psi \in \mathcal{P} \backslash \Pi$ for which $E^{*}(\psi)$ is stable (Corollary 4.1) and other values $\psi \in \Pi$ for which stability switch can occur (the local stability of $E^{*}$ is unknown for these values). The following proposition is adapted from Theorem 2.1 and 3.1 of Beretta and Kuang (2002).

Proposition 4.6. Assume that model parameters (different from $\psi$ ) are fixed and such that $\Pi \neq \emptyset$. The characteristic equation (12) admits a pair of simple conjugate purely imaginary roots $\pm \mathrm{i} \omega\left(\psi^{*}\right)$ in $\psi^{*} \in \Pi$ with

$$
\omega\left(\psi^{*}\right)=\sqrt{L\left(\psi^{*}\right)^{2}-\delta_{e}^{2}},
$$

if and only if there exists $k \in \mathbb{N}$ such that $z\left(\psi^{*}, k\right)=0$ with

$$
z(\psi, k)=\tau-\frac{\arctan \left(-\frac{\sqrt{L(\psi)^{2}-\delta_{e}^{2}}}{\delta_{e}}\right)+(2 k+1) \pi}{\sqrt{L(\psi)^{2}-\delta_{e}^{2}}},(\psi, k) \in \Pi \times \mathbb{N} .
$$

Moreover, when a boundary value $\psi^{*} \in \Pi$ exists and is reached due to a variation of $\psi$, its associated pair of simple conjugate purely imaginary roots cross the imaginary axis - possibly inducing a stability switch - from left to right if $\Delta\left(\psi^{*}\right)>0$ and from right to left if $\Delta\left(\psi^{*}\right)<0$ where

$$
\Delta\left(\psi^{*}\right)=\operatorname{sign}\left\{\frac{\mathrm{d}(\operatorname{Re} \lambda)}{\mathrm{d} \psi}\left(\psi^{*}\right)\right\} .
$$


This Proposition is proven in Appendix 3.

Remark 2. For given parameter values under which $\Pi \neq \emptyset$, a stability switch is possible only if there exists $k \in \mathbb{N}$ such that $z(\cdot, k)$ vanish at least one time.

Remark 3. When the chosen parameter varies from a value $\psi_{s} \notin \Pi$ for which $E^{*}\left(\psi_{s}\right)$ is stable, a Hopf bifurcation must occur at the first boundary value $\psi_{h}^{*}=\min \left\{\psi^{*} \mid\right.$ there exists $k \in \mathbb{N}$ such that $\left.z\left(\psi^{*}, k\right)=0\right\}$ if the transversality condition $\frac{\mathrm{d}(\operatorname{Re} \lambda)}{\mathrm{d} \psi}\left(\psi_{h}^{*}\right) \neq 0$ is verified.

Explicit form of $\frac{\mathrm{d}(\operatorname{Re} \lambda)}{\mathrm{d} \psi}$ is obtained by differentiating the characteristic equation (12) following the branch of roots $\lambda(\psi)$ defined such that $\lambda\left(\psi^{*}\right)=\mathrm{i} \omega\left(\psi^{*}\right)=\mathrm{i} \sqrt{L\left(\psi^{*}\right)^{2}-\delta_{e}^{2}}$. After some computations, one obtains :

$$
\begin{gathered}
\frac{\mathrm{d}(\operatorname{Re} \lambda)}{\mathrm{d} \delta_{e}}\left(\delta_{e}^{*}\right)=\frac{\partial L}{\partial \delta_{e}}\left(\delta_{e}^{*}\right) \frac{\sin \left(\omega\left(\delta_{e}^{*}\right) \tau\right) \omega\left(\delta_{e}^{*}\right) \tau-\cos \left(\omega\left(\delta_{e}^{*}\right) \tau\right)\left(1+\tau \delta_{e}^{*}\right)}{\left(1+\tau \delta_{e}^{*}\right)^{2}+\left(\omega\left(\delta_{e}^{*}\right) \tau\right)^{2}} \\
-\frac{\left(1+\tau \delta_{e}^{*}\right)}{\left(1+\tau \delta_{e}^{*}\right)^{2}+\left(\omega\left(\delta_{e}^{*}\right) \tau\right)^{2}}, \\
\frac{\mathrm{d}(\operatorname{Re} \lambda)}{\mathrm{d} K_{c}}\left(K_{c}^{*}\right)=\frac{\partial L}{\partial K_{c}}\left(K_{c}^{*}\right) \frac{\sin \left(\omega\left(K_{c}^{*}\right) \tau\right) \omega\left(K_{c}^{*}\right) \tau-\left(1+\tau \delta_{e}\right) \cos \left(\omega\left(K_{c}^{*}\right) \tau\right)}{\left(1+\tau \delta_{e}\right)^{2}+\left(\omega\left(K_{c}^{*}\right) \tau\right)^{2}}, \\
\frac{\mathrm{d}(\operatorname{Re} \lambda)}{\mathrm{d} \delta_{m e}}\left(\delta_{m e}^{*}\right)=\frac{\partial L}{\partial \delta_{m e}}\left(\delta_{m e}^{*}\right) \frac{\sin \left(\omega\left(\delta_{m e}^{*}\right) \tau\right) \omega\left(\delta_{m e}^{*}\right) \tau-\cos \left(\omega\left(\delta_{m e}^{*}\right) \tau\right)\left(1+\tau \delta_{e}\right)}{\left(1+\tau \delta_{e}\right)^{2}+\left(\omega\left(\delta_{m e}^{*}\right) \tau\right)^{2}}, \\
\frac{\mathrm{d}(\operatorname{Re} \lambda)}{\mathrm{d} \tau}\left(\tau^{*}\right)=\frac{\partial L}{\partial \tau}\left(\tau^{*}\right) \frac{\omega\left(\tau^{*}\right)^{3} \tau^{*} \sin \left(\omega\left(\tau^{*}\right) \tau^{*}\right)}{\left(1+\tau^{*} \delta_{e}\right)^{2}+\left(\omega\left(\tau^{*}\right) \tau^{*}\right)^{2}} \\
-\frac{\cos \left(\omega\left(\tau^{*}\right) \tau^{*}\right)\left(1+\tau^{*} \delta_{e}\right)}{\left(1+\tau^{*} \delta_{e}\right)^{2}+\left(\omega\left(\tau^{*}\right) \tau^{*}\right)^{2}} .
\end{gathered}
$$

In Fig. 2 and Fig. 3, we display stability diagrams in the $\left(\tau, \delta_{e}\right)$-plane (Fig. 2a and Fig. 3c), in the $\left(K_{c}, \delta_{e}\right)$-plane (Fig. 2c) and in the $\left(\delta_{e}, \delta_{m e}\right)$-plane (Fig. 3a) as well as example trajectories (Fig. 2b, Fig. 2d, Fig. 3b and Fig. 3d) corresponding to values specified by crosses (respectively in Figs. 2a, $2 \mathrm{c}, 3 \mathrm{a}$ and $3 \mathrm{c}$ ). The parameters used to obtain these plots are set to values mentioned in Table 2 . In Fig. 2d, Fig. 3b and Fig. 3d the red blood cell death rate is set to an arbitrary unrealistic value $\delta_{e}=0,07$ day $^{-1}$ for illustrative purpose.

Note. Asymptotic solutions turned out to be independent from initial conditions and densities. We thus arbitrarily decided to compute each trajectories showed in Fig. 3d, Fig. 2b, Fig. 2d and Fig. 3b with an initial condition and densities corresponding to $80 \%$ of the associated steady state specified by parameter values.

\section{Application to periodic oscillations of erythrocytes}

Let us first apply our model to experiments carried out on rabbits (Section 5.1) and check that our model is able to accurately describe periodic oscillations specific to the erythrocytic lineage.

\subsection{In rabbits}

We further assess the relevance of our model by comparing its output with experimental measurements of Orr et al. (1968). In their study, the authors regularly administrated (every 2 or 3 days) red blood cell antibodies to rabbits and consequently induced an immune hemolytic anemia. In the subject presented in the figure 4 of Orr et al. (1968), we observe reticulocyte and hemoglobin oscillations around 75 per cent of the normal level with a period of 16 to 17 days and an amplitude of approximately $10 \%$. To compare our modeling with these experimental results, we carefully choose model parameters displayed in Table 3. To do so, we follow experimental observations and statements of Orr et al. (1968) 


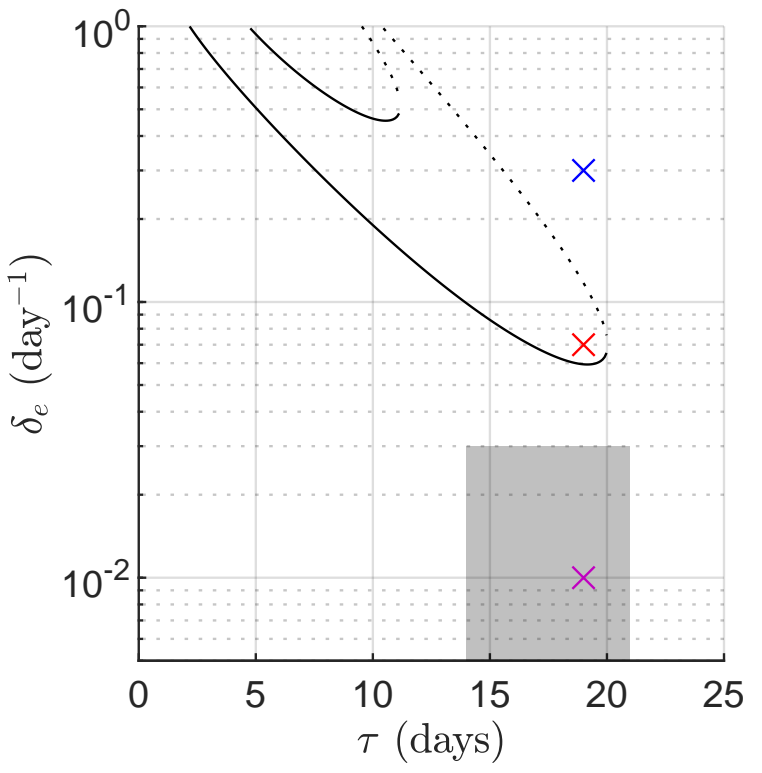

(a)

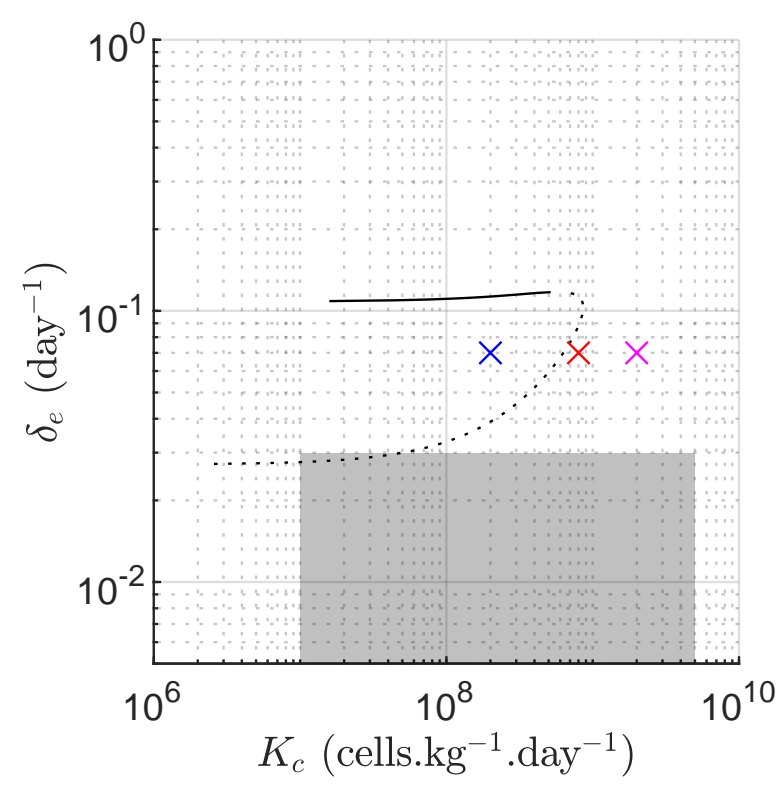

(c)

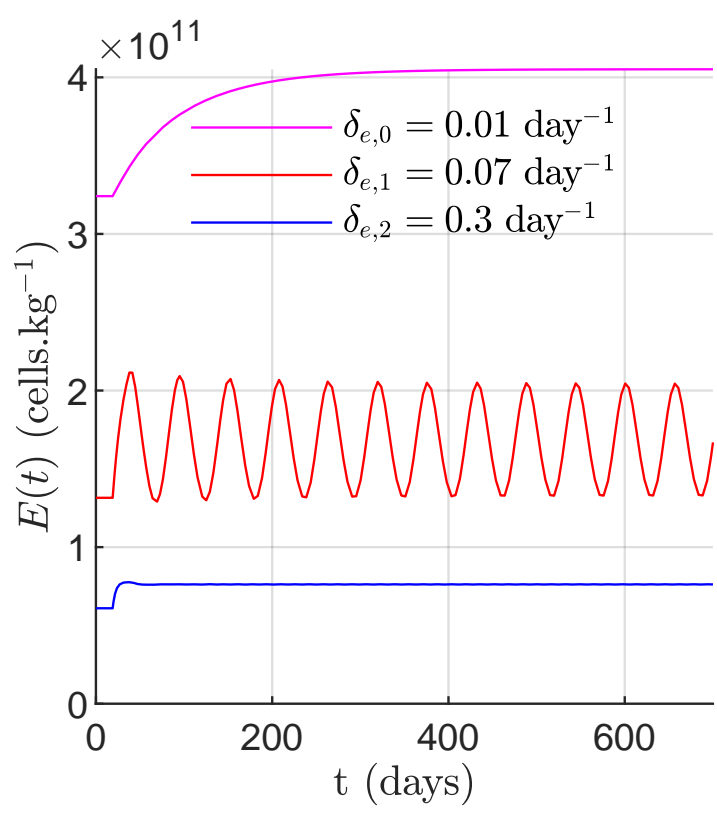

(b)

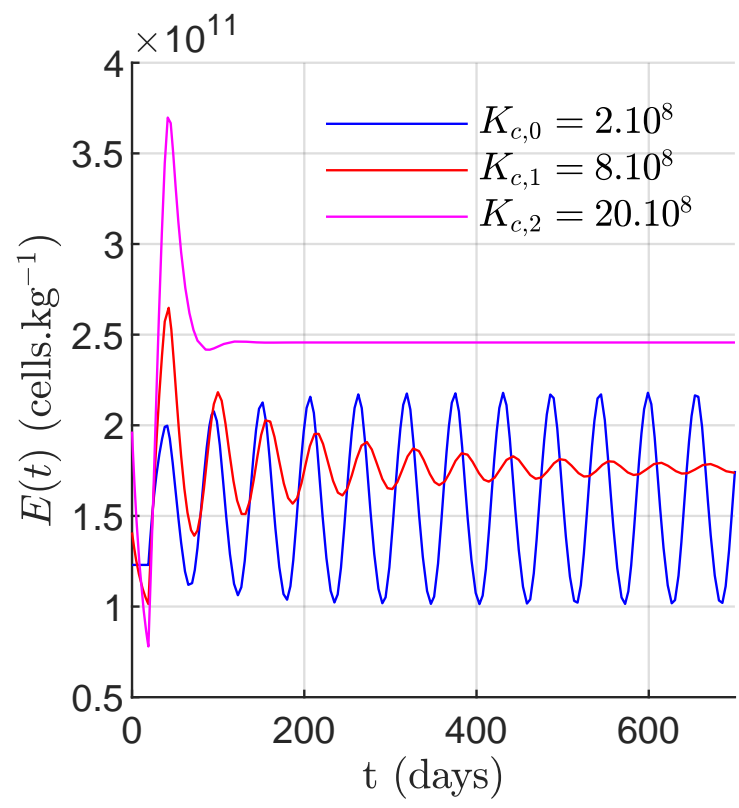

(d)

Figure 2 On the left panel: stability diagrams where boundary parameters $\left((\mathbf{a}) \psi^{*}=\delta_{e}^{*}\right.$ for red blood cell death rate, $(\mathbf{c}) \psi^{*}=K_{c}^{*}$ for hematopoietic stem cells influx) are specified by dashed $\left(\frac{\mathrm{d}(\operatorname{Re} \lambda)}{\mathrm{d} \psi}\left(\psi^{*}\right)<0\right)$ or full $\left(\frac{\mathrm{d}(\operatorname{Re} \lambda)}{\mathrm{d} \psi}\left(\psi^{*}\right)>0\right)$ lines. The gray zone corresponds to a biologically relevant area. On the right panel: illustrative trajectories associated to different erythrocyte death rate $((\mathbf{b})$ for values indicated by a cross in (a)) or different hematopoietic stem cells influx ((d) for values in cells. $\mathrm{kg}^{-1} \cdot \mathrm{day}^{-1}$ indicated by crosses in (c)). The parameters used to obtain these plots are specified in Table 2 . 


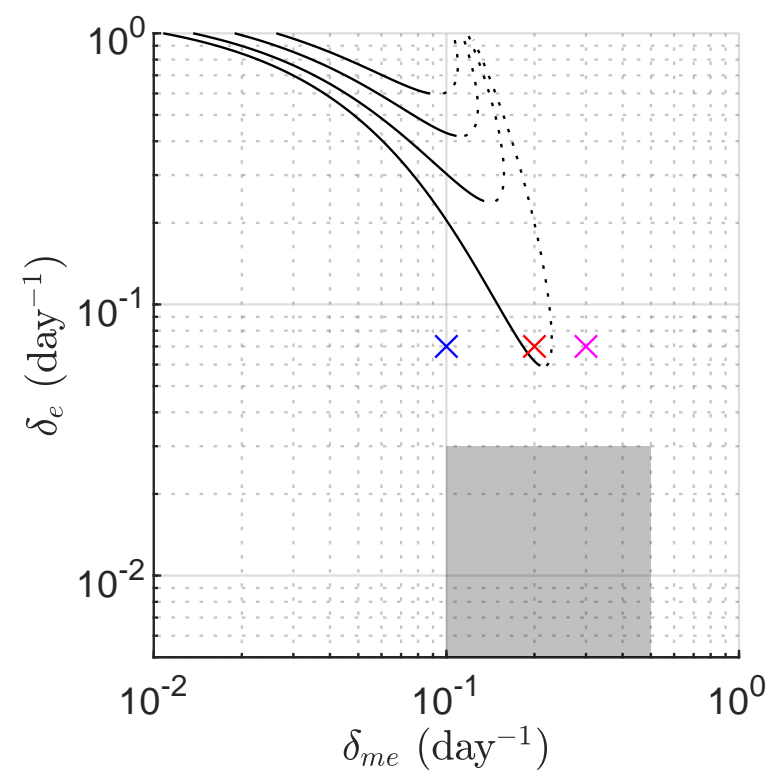

(a)

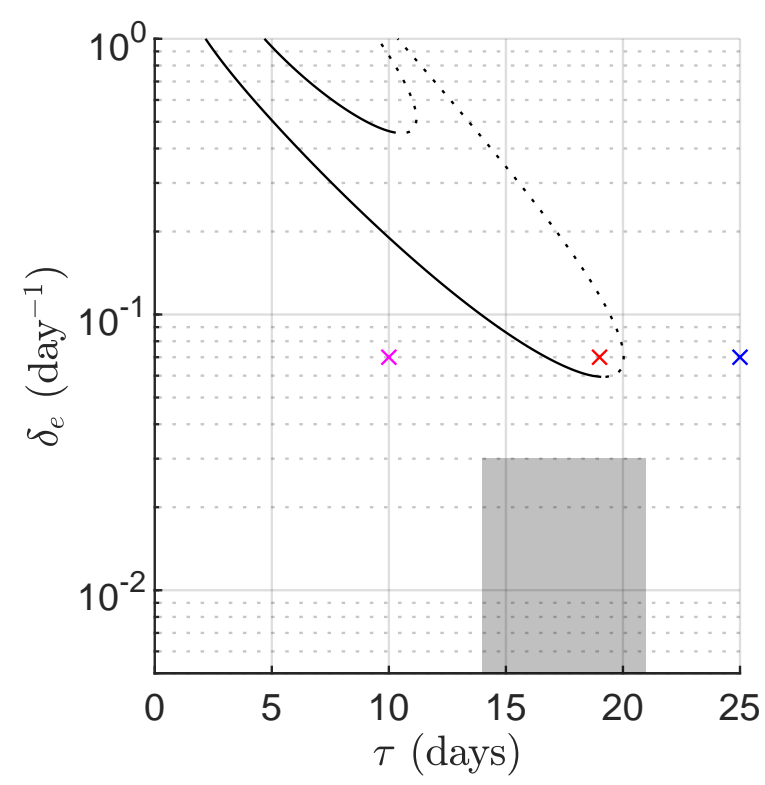

(c)

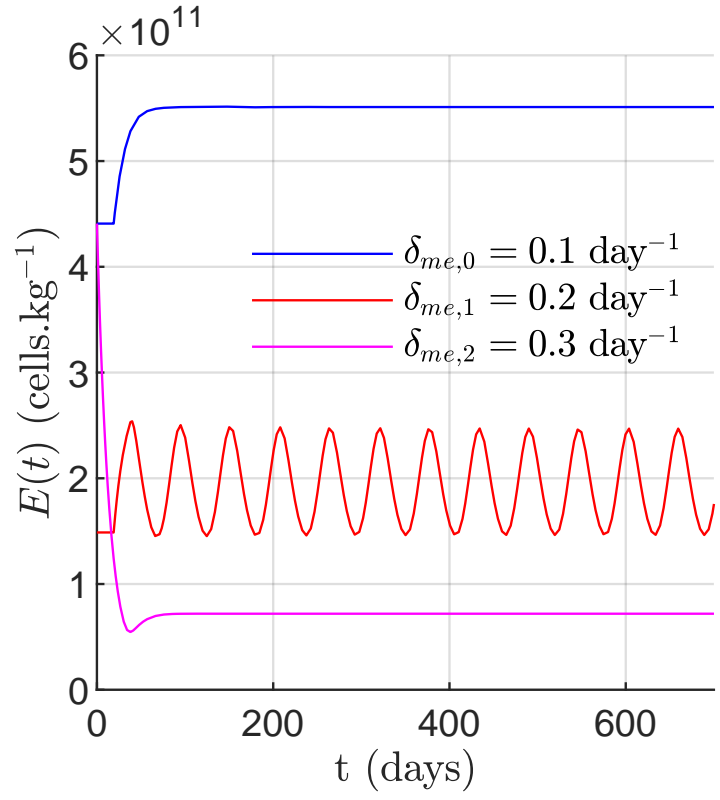

(b)

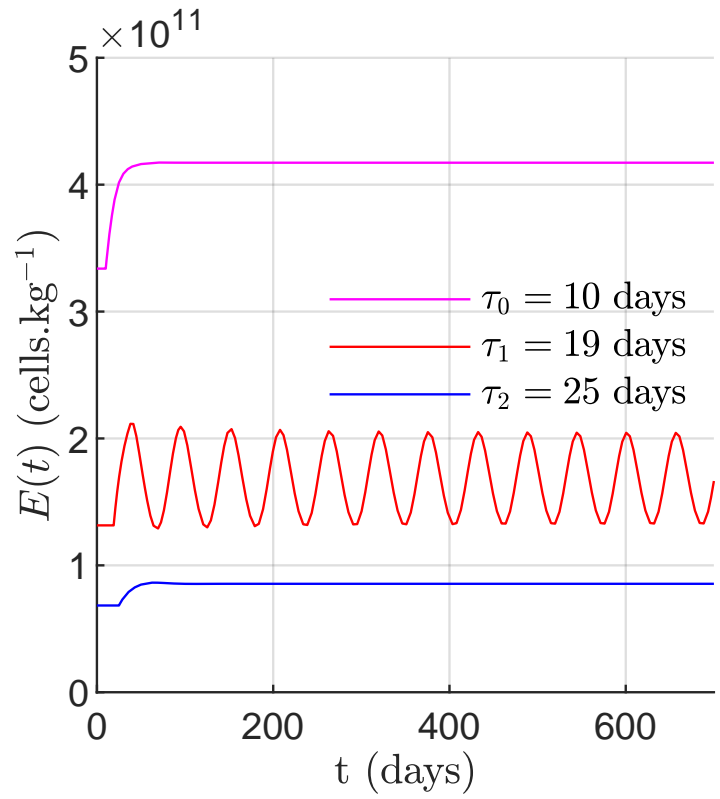

(d)

Figure 3 On the left panel: stability diagrams where boundary parameters $\left(\mathbf{( a )} \psi^{*}=\delta_{m e}^{*}\right.$ for bone marrow cells death rate; (c) $\psi^{*}=\tau^{*}$ for bone marrow cells transit time) are specified by dashed $\left(\frac{\mathrm{d}(\operatorname{Re} \lambda)}{\mathrm{d} \psi}\left(\psi^{*}\right)<0\right)$ or full $\left(\frac{\mathrm{d}(\operatorname{Re} \lambda)}{\mathrm{d} \psi}\left(\psi^{*}\right)>0\right)$ lines. They gray zone corresponds to a biologically relevant area. On the right panel: illustrative trajectories associated to example bone marrow cells death rates ((b) for values indicated by crosses in (a)) or example bone morrow transit time ((d) for values indicated by crosses in (c)). The parameters used to obtain these plots are specified in Table 2 
Table 2 Summary of model parameter values used in numeric simulations. Exact or approximated values from literature are used for ranges

\begin{tabular}{|c|c|c|c|}
\hline Parameter & Value assigned & Range & Unit \\
\hline$\tau$ & $\begin{array}{l}19 \text { or varying (only in } \\
\text { Figs. 3c, 3d) }\end{array}$ & {$[14,21]$} & days \\
\hline$\delta_{m e}$ & $\begin{array}{l}0,22 \text { or varying (only in } \\
\text { Figs. } 3 \mathrm{a}, 3 \mathrm{~b} \text { ) }\end{array}$ & {$[0,1,1]$} & day $^{-1}$ \\
\hline$\delta_{e}$ & $\begin{array}{l}0,07 \text { or varying (only in } \\
\text { Fig. } 2 \mathrm{a}, 2 \mathrm{~b} \text { ) }\end{array}$ & {$[0,01,0,03]$} & day $^{-1}$ \\
\hline$T_{e}$ & Not assigned & {$[40,120]$} & days \\
\hline $\begin{array}{l}\delta_{e, b} \\
A=2^{n}\end{array}$ & $\begin{array}{l}\text { Not assigned } \\
2^{9}\end{array}$ & $\begin{array}{l}{[0,001,0,01]} \\
{\left[2^{5}, 2^{11}\right]}\end{array}$ & day $^{-1}$ \\
\hline$K_{c}$ & $\begin{array}{l}5.10^{8} \text { or varying (only in } \\
\text { Figs. } 2 \mathrm{c}, 2 \mathrm{~d} \text { ) }\end{array}$ & {$\left[10^{7}, 5.10^{9}\right]$} & cells. $\mathrm{kg}^{-1}$.day $^{-1}$ \\
\hline$\alpha$ & $\begin{array}{l}25.10^{8}\left(=5 K_{c} \text { excepted }\right. \\
\text { when varying in Figs. } 2 \mathrm{c} \text {, } \\
2 \mathrm{~d})\end{array}$ & {$\left[3 K_{c}, 6 K_{c}\right]$} & ${\text { cells. } \mathrm{kg}^{-1} \text {. day }}^{-1}$ \\
\hline$\beta$ & $1,5.10^{11}$ & approx. $10^{11}$ & cells. $\mathrm{kg}^{-1}$ \\
\hline$q$ & 5 & - & - \\
\hline
\end{tabular}

as well as the parameter choice approach of Mackey (1979); Bélair et al. (1995) and Mahaffy et al. (1998). Firstly, we set the initial red blood cell level to the average normal level for humans (Beutler et al., 2001): $E_{0}=3,5 \times 10^{11}$ cells.kg ${ }^{-1}$. Parameters $A, n$ and $K_{c}$ are chosen within range observed in mammals (see e.g. Crauste et al., 2008; Greer et al., 2003). Given the lack of data, we decide to arbitrarily choose the values of $q$ and $\alpha$ such that the model output present periodic oscillations. Setting $q=18$ and $\alpha=8 K_{c}$ (it remains in a plausible range) leads to consider that feedback processes are fast, our choices may thus be unrealistic. Then, we set the value of $\beta$ such that it verifies the assertion of Orr et al. (1968) declaring that "a reduction of the average hemoglobin level to about 75 per cent of its normal value has increased differentiation by a factor of at least 5". We also choose $\tau$ arbitrarily but still in a range of meaningful values. Actually, Orr et al. (1968) wrote: "the time spent in the erythron cannot be more than 3 days". However, this ambiguous assertion does not clearly define which cell stages are designated through the term "erythron". One especially ignores if $B F U-E$ and $C F U-E$ are considered or not. Hence, the marrow transit time in our modeling is likely to be greater than 3 days and the choice of $\tau=8$ days is plausible. The mean lifespan of erythrocytes is chosen according to the measurements of Orr et al. (1968), that is $T_{e}=14$ days and consequently (see Eq. (4)) $\delta_{e}=0,0785$ day $^{-1}$. We adapted the choice of $\delta_{m e}$ in order to obtain a solution who oscillate around 75 per cent of its normal level. Lastly, the initial erythrocyte death rate $\delta_{e, 0}$ (and thus red blood cell lifespan $\left.T_{g b, 0}\right)$ are imposed by these choices ensuring that the initial equilibrium - $E_{0^{-}}$is well defined. We obtain $\delta_{e, 0}=0,0122$ day $^{-1}$ corresponding to $T_{g b, 0} \simeq 90$ days which is significantly higher than normal values of 45 to 50 days found in literature (Burwell et al., 1953). Initial population densities are steady state populations given in Eq. (10) associated to $E_{0}$.

Positions of the initial situation (normal with $\delta_{e, 0}$ ) and the experimental one (induced immune hemolytic anemia with $\left.\delta_{e}\right)$ in the $\left(\tau, \delta_{e}\right)$-plane as well as boundary of stability regions $\left(\delta_{e}^{*}\right)$ are illustrated in Fig. 4a. The model solution displayed in Fig. 4b periodically oscillates around an unstable equilibrium of $0,75 E_{0, \text { init }}$ with a mean periodicity of 16,9 days and an amplitude of $13,6 \%$. Although parameter choices impose an unrealistic normal mean lifespan of erythrocyte, our model output is not far from the observations shown in the figure 4 of Orr et al. (1968). The application to rabbits seems to support the relevance of our model to describe periodic diseases specific to the erythrocyte lineage in other species and especially humans. 
Table 3 Parameter values used for model simulation of the immune hemolytic anemia experiment in rabbits. Exact or approximated values from literature are used for ranges

\begin{tabular}{llll}
\hline Parameter & Value assigned & Range & Unit \\
\hline$\tau$ & 5 & {$[14,21]$} & days \\
$\delta_{m e}$ & 0,2286 & {$[0,1,1]$} & day $^{-1}$ \\
$\delta_{e}$ & 0,0785 or 0,0122 & {$[0,01,0,03]$} & day $^{-1}$ \\
$T_{e}$ & 14 or 90,1 & {$[7,50]$} & days \\
$\delta_{e, b}$ & Not assigned & {$[0,001,0,01]$} & day $^{-1}$ \\
$n$ & 7 & {$[5,11]$} & - \\
$A=2^{n}$ & $2^{7}$ & {$\left[2^{5}, 2^{11}\right]$} & - \\
$K_{c}$ & $1.10^{8}$ & {$\left[10^{7}, 5 \cdot 10^{9}\right]$} & cells.kg $^{-1} \cdot$ day $^{-1}$ \\
$\alpha$ & $8.10^{8}\left(=8 K_{c}\right)$ & {$\left[3 K_{c}, 6 K_{c}\right]$} & cells.kg $^{-1} \cdot$ day $^{-1}$ \\
$\beta$ & $2,6283.10^{11}$ & approx. $10^{11}$ & cells.kg \\
$q$ & 18 & - & - \\
\hline
\end{tabular}

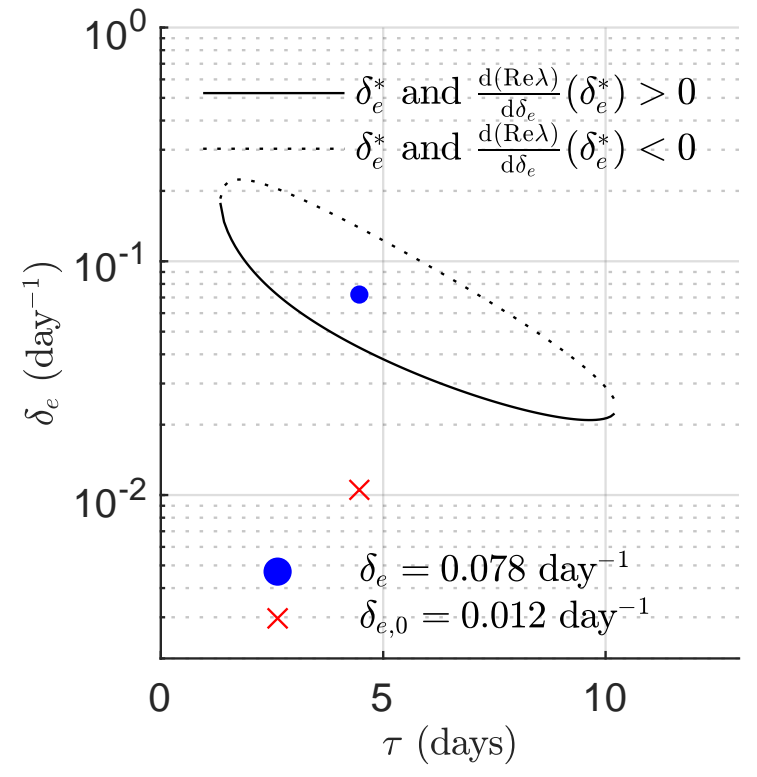

(a)

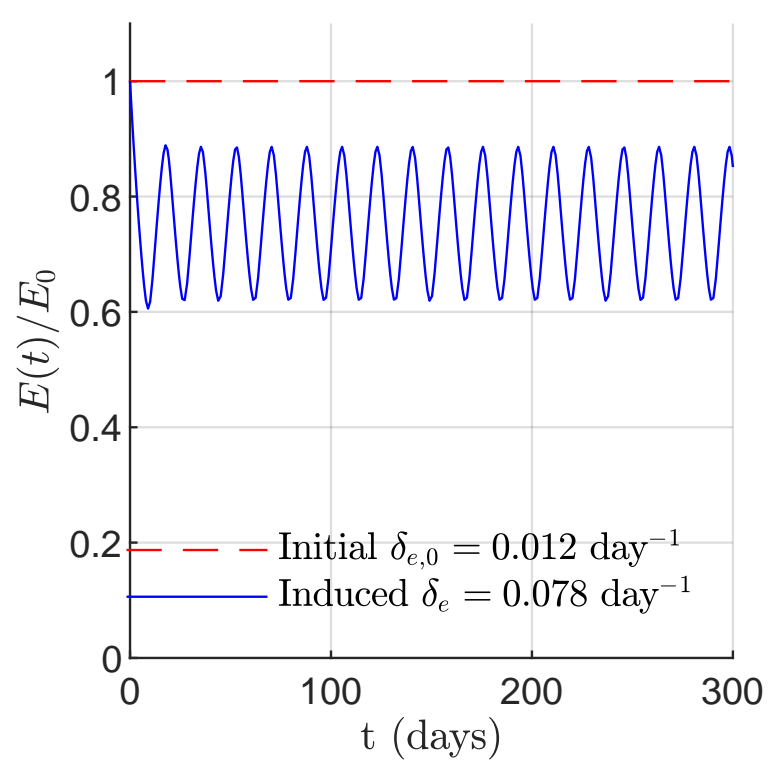

(b)

Figure 4 (a) Stability diagram obtained in the framework of Orr et al. (1968) experiment. (b) Model output $\frac{E(t)}{E_{0}}$ associated to model parameters specified in Table 3 


\subsection{In humans}

In Fig. 2 and Fig. 3, we considered the case study of a human subject and paid particular attention to set fixed parameters to physiologically meaningful values (see Table 2, excepted for $\delta_{e}$ which has been set to $\delta_{e}=0.07$ day $^{-1}$ for illustrative purpose in Fig. 3d, Fig. 2d and Fig. 3b). We only have uncertainties about the biological relevance of the values taken for $K_{c}, \alpha, \beta$ and $q$. Because of a lack of clearly referenced data for these parameters, we set them similarly to what is usually done in other modeling studies specific to erythropoiesis (see e.g. Mackey, 1979; Bélair et al., 1995; Crauste et al., 2008). Using ranges of parameter values referenced in Table 1, we identify physiologically meaningful regions of parameter space (which include extreme values seen in some diseases) with coloured areas in Fig. 3c, Fig. 2a, Fig. 2c and Fig. 3a. It is important to note here that these biologically relevant regions predominantly lie in regions where $E^{*}$ is locally asymptotically stable consequently excluding the possibility of oscillations under physiologically conceivable conditions. In the case of the variation of $\tau, \delta_{e}$ and $\delta_{m e}$ (Fig. 3c, Fig. 2a and Fig. 3a), we underline that biologically relevant areas do not include stability switch boundaries and are thus disjoint from unstable regions. Consequently, it seems that a variation of these parameters under biologically conceivable conditions is highly unlikely to lead to periodic fluctuations of red blood cell levels. When it comes to the parameter $K_{c}$ (basal influx of hematopoietic stem cells) in Fig. 2c, the interpretation is almost the same excepted that the biologically relevant area slightly encounter a stability boundary at very high erythrocyte death rates $\delta_{e}$ and low values of $K_{c}$. Consequently $K_{c}$ may be the only parameter which might induce periodic fluctuations specific to erythrocytes in humans. This is in agreement with the observation of Gordon and Varadi (1962) in which erythrocytic specific oscillations are due to bone marrow defects. Moreover, $K_{c}$ being tightly associated with the hematopoietic stem cell population (whose dynamics is not considered in this paper), our analysis also seems to support other studies (see e.g. Haurie et al., 1998; Mackey, 1997) in which erythrocytic oscillations would originate from the hematopoietic stem cell population itself and not from erythropoiesis.

All in all, thanks to literature data and our analysis, one can understand why periodic hematological diseases specific to erythrocytes are so rarely observed. We state that human erythropoiesis should operate in parameter regions where periodic red blood cell oscillations cannot occur even under pathological conditions. Actually periodic fluctuations specific to red blood cell lineage are highly unlikely to appear under physiologically possible parameters. However, if it is the case, it might be due to an abnormally low value of basal hematopoietic stem cell influx and/or a high red blood cell death rate.

\section{Further improvements of the model}

We suggest to improve the previous model into the one illustrated in Fig. 5. However, due to its complexity, it can only be studied numerically and this is the main reason why we give it only in this last section. Modifications of the first model are the following:

- the population of bone marrow cells is divided in two: the bone marrow nucleated cells with a transit time $T_{p}$ and a death rate $\delta_{p}$ and the (unnucleated) Marrow reticulocytes with a transit time $T_{r}$ and a death rate $\delta_{r}$. As mentioned in Section 2.1 as a limit of the simple model presented in Fig.1, EPO here not only affects hematopoietic stem cell influx, but also the apoptosis rate $\delta_{p}$ of erythroid progenitors $(C F U-E)$. In a standard way, we use an increasing Hill function to describe this feedback:

$$
\delta_{p}(E)=\delta_{p, \min }+\left(\delta_{p, \max }-\delta_{p, \min }\right) \frac{E^{q_{b}}}{E^{q_{b}}+\beta_{p}^{q_{b}}}, E \geq 0,
$$

where $\delta_{p, \min }$ and $\delta_{p, \max }$ account respectively for the minimal and maximal death rate of bone marrow nucleated cells. $\beta_{p}>0$ is the total number of erythrocytes corresponding to the median death rate $\frac{\delta_{p, \min }+\delta_{p, \max }}{2}$ and $q_{b} \geq 1$ is the Hill coefficient characterizing how fast is the change between red blood cells number and progenitor apoptosis. All things considered, similar to the 


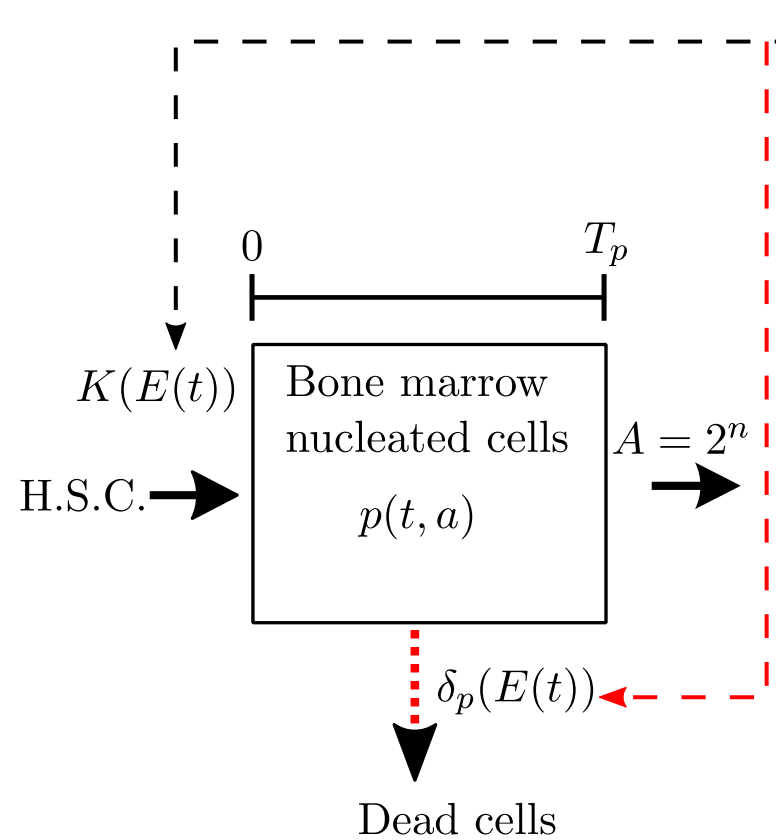

Bone Marrow

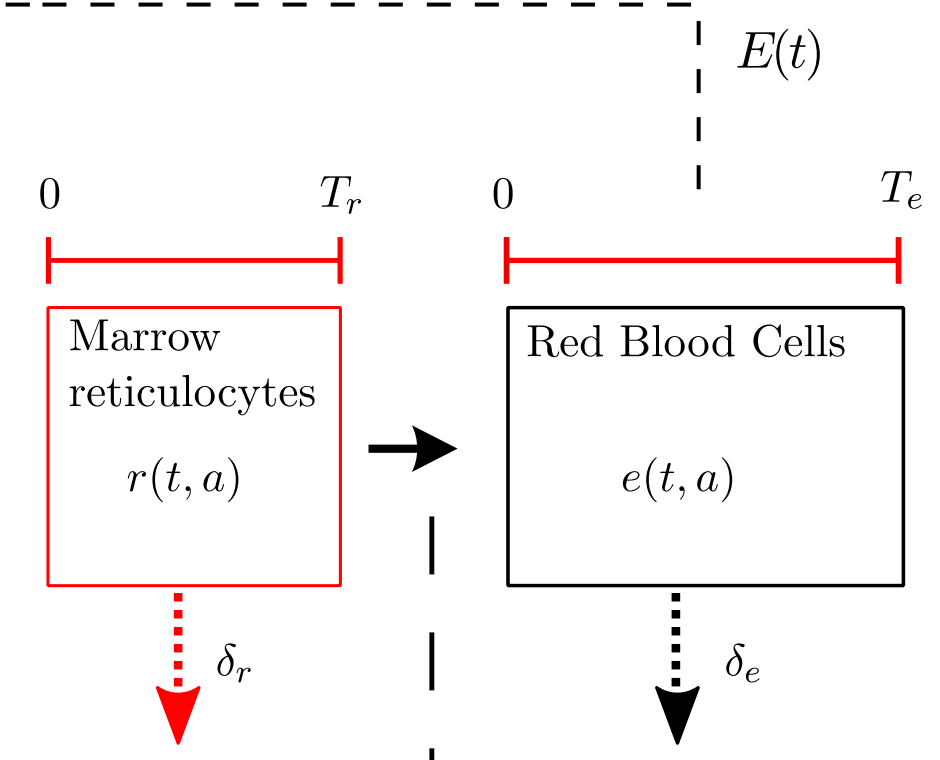

Dead cells

Dead cells

Peripheral Blood

Figure 5 Erythropoiesis model improved. Main modifications of the last model are indicated in red. Previously described bone marrow population is now divided in two : the bone marrow nucleated cells and the Marrow reticulocytes. The finite transit time in cell compartments are $T_{p}$ for bone marrow nucleated cells, $T_{r}$ for marrow reticulocytes and $T_{e}$ for red blood cell. Population death rates are $\delta_{p}$, $\delta_{r}$ and $\delta_{e} . A=2^{n}$ translates the $n \in \mathbb{N}$ mitotic divisions underwent by a single nucleated cell during its development in the bone marrow. This value may be a little less if we take cell apoptosis in every doubling stage. This is why we assume that $A$ belongs in the interval $\left[2^{5}, 2^{11}\right]$ in Table 4 . Total red blood cell population - $E$ - downregulates erythropoiesis through a negative feedback over the cell influx $K(E)$ and through a positive feedback over the death rate of bone marrow nucleated cells $\delta_{p}(E)$. 
previous modeling, corresponding densities are also ruled by transport equations:

$$
\begin{gathered}
\frac{\partial p}{\partial t}(t, a)+\frac{\partial p}{\partial a}(t, a)=-\delta_{p}(E(t)) p(t, a), t \geq 0, a \in\left[0, T_{p}\right], \\
\frac{\partial r}{\partial t}(t, a)+\frac{\partial r}{\partial a}(t, a)=-\delta_{r} r(t, a), t \geq 0, a \in\left[0, T_{r}\right] .
\end{gathered}
$$

- Cells cannot transit infinitely in the red blood cell compartment, we now consider a finite transit time $T_{e}$ accounting for the finite lifespan of erythrocytes. Each red blood cell reaching age $T_{e}$ is identified as senescent by macrophages and is actively degraded due to phagocytosis. In this paradigm $\delta_{e}$ no longer accounts for senescence (Eq. (4) does not hold anymore) and its meaning becomes more consistent with its unspecific nature. $\delta_{e}$ only models other causes of red blood cell destruction that are -in the vast majority- random and non specific. We use the same framework to describe the erythrocyte density $e$ excepted concerning the finite lifespan:

$$
\frac{\partial e}{\partial t}(t, a)+\frac{\partial e}{\partial a}(t, a)=-\delta_{e} e(t, a), t \geq 0, a \in\left[0, T_{e}\right] .
$$

Total populations obtained after integration of the equations (18), (19) and (20) over the corresponding age intervals are suitable to accurately model erythropoiesis. Derivation of the corresponding model equations is not the aim of this work, we simply state that solution dynamics at long time $t \geq$ $T_{e}+T_{r}+T_{p}$ is entirely ruled by

$$
\begin{array}{r}
E^{\prime}(t)=-\delta_{e} E(t)+A \mathrm{e}^{-T_{r} \delta_{r}}\left[K\left(E\left(t-\left(T_{r}+T_{p}\right)\right)\right) \mathrm{e}^{-\int_{T_{r}}^{T_{r}+T_{p}} \delta_{p}(E(t-u)) \mathrm{d} u}\right. \\
\left.-\mathrm{e}^{-T_{e} \delta_{e}} K\left(E\left(t-T_{e}-\left(T_{r}+T_{p}\right)\right)\right) \mathrm{e}^{-\int_{T_{e}+T_{r}}^{T_{e}+T_{r}+T_{p}} \delta_{p}(E(t-u)) \mathrm{d} u}\right] .
\end{array}
$$

This non-linear delay differential equation admits a unique positive equilibrium $E^{*}>0$ solving

$$
\delta_{e} E^{*}=A\left(1-\mathrm{e}^{-T_{e} \delta_{e}}\right) K\left(E^{*}\right) \mathrm{e}^{-T_{r} \delta_{r}-T_{p} \delta_{p}\left(E^{*}\right)} .
$$

However, Eq. (21) involves discrete and distributed delays which render the local asymptotic study of solutions around $E^{*}$ particularly tedious and kept for a future work. The resulting characteristic equation is

$$
\lambda+\delta_{e}+\mathrm{e}^{-\lambda T_{r}}\left[1-\mathrm{e}^{-\left(\lambda+\delta_{e}\right) T_{e}}\right]\left[\xi_{1}\left(\int_{0}^{T_{p}} \mathrm{e}^{-\lambda u} \mathrm{~d} u\right)-\xi_{2} \mathrm{e}^{-\lambda T_{p}}\right]=0,
$$

with

$$
\begin{aligned}
\xi_{1} & =A \mathrm{e}^{-T_{r} \delta_{r}-T_{p} \delta_{p}\left(E^{*}\right)} K\left(E^{*}\right) \frac{\partial \delta_{p}}{\partial E}\left(E^{*}\right), \\
\xi_{2} & =A \mathrm{e}^{-T_{r} \delta_{r}-T_{p} \delta_{p}\left(E^{*}\right)} \frac{\partial K}{\partial E}\left(E^{*}\right) .
\end{aligned}
$$

One would remark that 0 cannot be a root of the latter equation due to the variations of $K$ and $\delta_{p}$ with respect to $E$. Consequently, the characteristic equation of Eq. (21) linearized about its unique positive equilibrium $E^{*}$ reads

$$
\lambda+\delta_{e}+\mathrm{e}^{-\lambda T_{r}}\left[1-\mathrm{e}^{-\left(\lambda+\delta_{e}\right) T_{e}}\right] \frac{\left[\xi_{1}-\left(\xi_{1}+\lambda \xi_{2}\right) \mathrm{e}^{-\lambda T_{p}}\right]}{\lambda}=0 .
$$

As mentioned above, finding the roots of Eq. (22) is an open problem and is only carried out numerically here. In Fig. 6a we provide an insight into the stability of the model in the $\left(T_{p}, T_{e}\right)$-plane. Thanks to a " $\tau$-decomposition" method, for a given value of $T_{p}$, we attempt to determine boundary red blood cell transit time at which stability switches might occur. Fixed model parameters are set to biologically 
Table 4 Parameter values used in Fig. 6 for the integration of Eq. (21). Exact or approximated values from literature are used for ranges

\begin{tabular}{|c|c|c|c|c|}
\hline Parameter & Value assigned & & Range & Unit \\
\hline$T_{p}$ & $\begin{array}{l}\text { varying } \\
\text { (Fig. 6b) }\end{array}$ (Fig. 6) & 19 & {$[12,20]$} & days \\
\hline$T_{r}$ & 1 & & {$[1,3]$} & days \\
\hline$\delta_{r}$ & 0.05 & & {$[0,1,1]$} & day $^{-1}$ \\
\hline$\delta_{e}$ & 0.005 & & {$[0,01,0,03]$} & day $^{-1}$ \\
\hline$T_{e}$ & varying & & {$[60,120]$} & days \\
\hline$n$ & 9 & & {$[5,11]$} & - \\
\hline$A=2^{n}$ & $2^{9}$ & & {$\left[2^{5}, 2^{11}\right]$} & - \\
\hline$K_{c}$ & $1.10^{8}$ & & {$\left[10^{7}, 5.10^{9}\right]$} & cells.kg ${ }^{-1}$. day $^{-1}$ \\
\hline$\alpha$ & $5.10^{8}\left(=5 K_{c}\right)$ & & {$\left[3 K_{c}, 6 K_{c}\right]$} & cells.kg ${ }^{-1}$. day $^{-1}$ \\
\hline$\beta$ & $3.3 .10^{11}$ & & approx. $10^{11}$ & cells. $\mathrm{kg}^{-1}$ \\
\hline$q$ & 8 & & - & - \\
\hline$\delta_{p, \min }$ & 0.01 & & - & day $^{-1}$ \\
\hline$\delta_{p, \max }$ & 0.3 & & - & day $^{-1}$ \\
\hline$\beta_{p}$ & $3.3 .10^{11}$ & & approx. $10^{11}$ & cells. $\mathrm{kg}^{-1}$ \\
\hline$q_{p}$ & 8 & & - & - \\
\hline
\end{tabular}

relevant values as specified in Table 4 . In Fig. $6 \mathrm{~b}$ we illustrate the stability switch by considering two situations with the same $T_{p}$ and two different red blood cell transit time: $T_{e, 1}$ and $T_{e, 2}$. Here, $T_{e, 1}$ represents the normal conditions (that is a 120 days lifespan for erythrocytes while $T_{e, 2}$ represents a critical pathological condition (parameter value given in Table 4, with reference given from Table 1. Thanks to the MATLAB solver dde23 we integrate Eq. (21) for the situations $i \in\{1,2\}$ assuming a blood loss at $t=0$ and $E_{i}(t)=E_{i}^{*}$ for all $t<0$. These examples and others (not shown here) highlight that solution dynamics is likely to be more complicated without being drastically different.

\section{Summary and conclusion}

We analyzed an erythropoiesis model and attempted to account for the rare observations of cyclic red blood cell oscillations. Motivated by recent experimental observations concerning the effect of EPO on the commitment of hematopoietic stem cells into the erythrocytic lineage, we provided a mathematical model of erythropoiesis generalizing the one in Mackey (1997). We continued and improved this work by adding a basal cell influx (parameter $K_{c}$ ) and by providing a more detailed mathematical and numerical analysis. We numerically obtained the boundaries of stability switches in parameter space. With the resulting stability diagrams and a thorough insight into erythropoiesis specific literature we completed the work of Mackey $(1979,1997)$. Two cases study enabled us to highlight key aspects involved in the occurrence of periodic erythrocytic oscillations. As pointed out by Mackey (1979), Bélair et al. (1995) and Mahaffy et al. (1998), our model also predicts that an increase in the erythrocyte death rate $\delta_{e}$ may trigger periodic oscillations of erythrocytes in rabbit. Our contribution lies in the identification, in the parameter space, of biologically relevant areas for humans from which we explained the extremely rare observations of cyclic erythropoiesis in humans. We particularly identified that human parameter values are responsible for the very sparse observations of periodic red blood cell levels and if observed, this periodicity might only be due to abnormally high erythrocyte death rate and/or abnormally low basal hematopoietic stem cells influx. Carefully inspecting erythropoiesis processes, we finally highlighted the limit of our model and developed an improved one. Its tedious characteristic equation and stability switches are still to be investigated. Moreover this new model grasps more biological aspects (especially concerning erythropoietin feedback over apoptosis of erythroid progenitor and red blood cell removal) while remaining simple with a reasonable number of parameters. Thus, added to a pharmacokinetic modeling of EPO and possibly a model of iron dynamics, it opens up prospects for hemoglobin regulation of dialysis patients. 


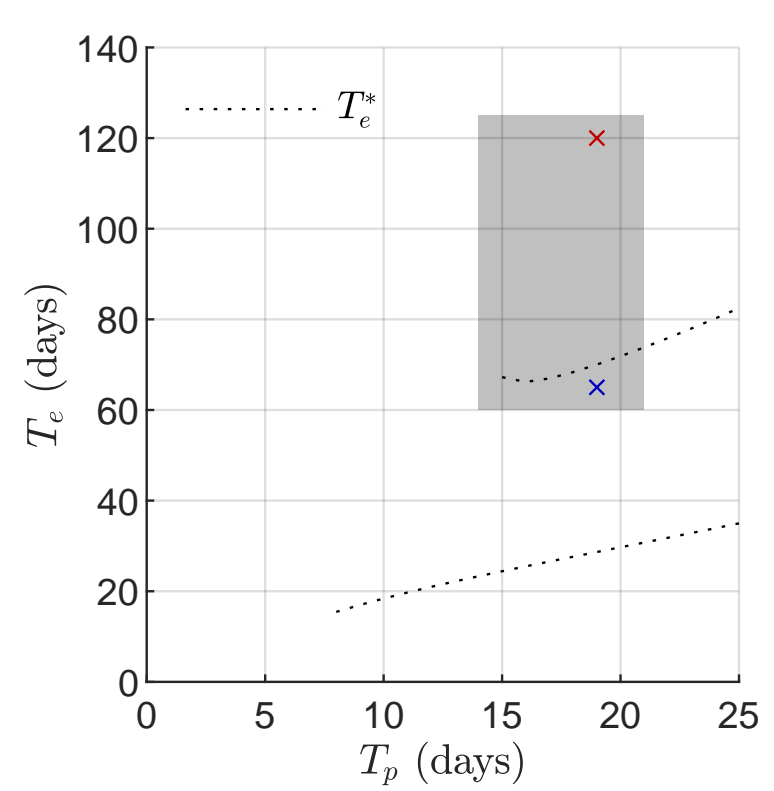

(a)

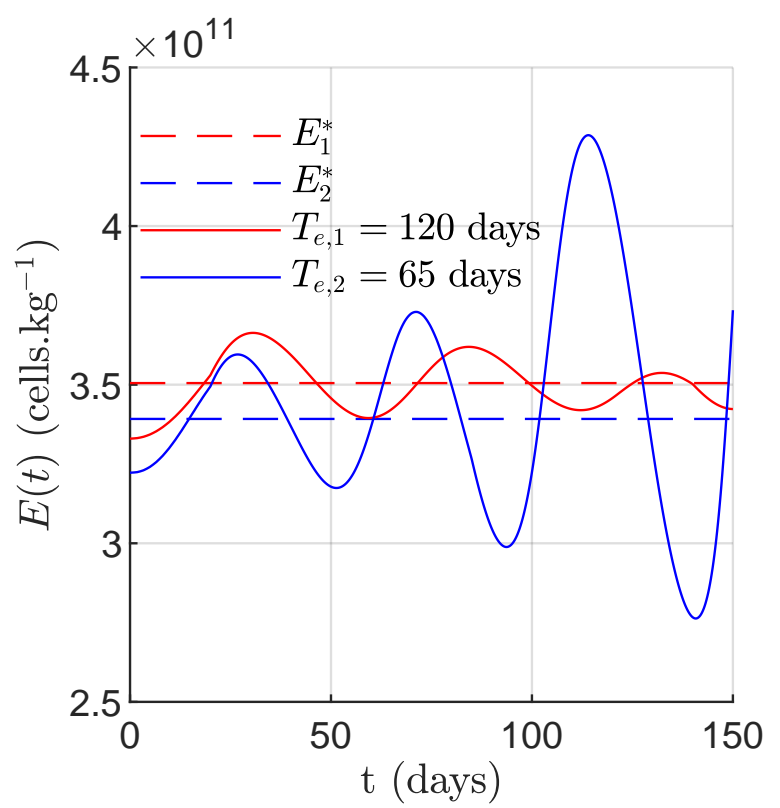

(b)

Figure 6 (a) Stability diagram of the improved model associated with the characteristic equation Eq. (22) in the $\left(T_{p}, T_{e}\right)$-plane. We remind that $T_{e, 1}$ is the parameter used to described normal conditions, and $T_{e, 2}$ a pathological one. A biologically relevant area is colored. (b) Illustrative trajectories associated to example red blood cell transit time specified in (a). The parameters used to obtain these plots are specified in Table 4

\section{Appendix 1: Sufficient stability condition}

In this appendix, we use the theorem established by Hayes (1950) to formulate a sufficient stability condition for the equilibrium $E^{*}$. Consider a set of model parameters $A, \delta_{m e}, q, \beta, K_{c}, \alpha, \delta_{e}$ and $\tau$. If $\tau=0$ then the characteristic equation (12) admits a unique root $\lambda=-\delta_{e}-L$ which is real and negative because $L>0$. Now, we consider that $\tau>0$. Eq. (12) can be written as

$$
\left(\lambda \tau+\delta_{e} \tau\right) e^{\lambda \tau}+L \tau=0, \lambda \in \mathbb{C} .
$$

The latter equation is adapted to the theorem formulated by Hayes (1950). Hence, if $\lambda \in \mathbb{C}$ verifies Eq. (23) then

$$
\operatorname{Re} \lambda<0 \Leftrightarrow\left\{\begin{array}{l}
0<L \tau<\mu \sin (\mu)-\delta_{e} \tau \cos (\mu), \\
\mu=-\delta_{e} \tau \tan (\mu), \mu \in\left(\frac{\pi}{2}, \pi\right) .
\end{array}\right.
$$

Which can also be written as

$$
\operatorname{Re} \lambda<0 \Leftrightarrow\left\{\begin{array}{l}
0<-\cos (\mu)<\frac{\delta_{e}}{L}, \\
\mu=-\delta_{e} \tau \tan (\mu), \mu \in\left(\frac{\pi}{2}, \pi\right) .
\end{array}\right.
$$

Given that $0<-\cos (\mu)<1$ holds for all $\mu \in\left(\frac{\pi}{2}, \pi\right)$, we obtain Proposition 4.1.

\section{Appendix 2: Continuity, differentiability and variations with respect to model parameters}

Derivatives with respect to $E$. Given the form of $K(\cdot)$ in Eq. (2), trivial derivation rules and simple computations lead, for $E>0$, to

$$
\frac{\partial K}{\partial E}(E)=-q \frac{1}{\beta}\left(\frac{E}{\beta}\right)^{q-1} \frac{\alpha}{\left(1+\left(\frac{E}{\beta}\right)^{q}\right)^{2}}>0,
$$


and

$$
\frac{\partial^{2} K}{\partial E^{2}}(E)=q \alpha \frac{(q+1)\left(\frac{E}{\beta}\right)^{2 q-2}-(q-1)\left(\frac{E}{\beta}\right)^{q-2}}{\beta^{2}\left(1+\left(\frac{E}{\beta}\right)^{q}\right)^{3}}
$$

Continuity and variations with respect to $\tau$. Remembering the fact that $L$ is strictly positive for all $\tau \geq 0$, one can establish from the implicit function theorem that, all other parameters being fixed, $\tau \mapsto E^{*}(\tau)$ is continuously differentiable on $[0,+\infty)$ and

$$
\frac{\partial E^{*}}{\partial \tau}(\tau)=\frac{-\delta_{m e} \delta_{e} E^{*}(\tau)}{\delta_{e}+L(\tau)}>0
$$

$\tau \mapsto E^{*}(\tau)$ is thus a strictly decreasing function with $\lim _{\tau \rightarrow \infty} E^{*}(\tau)=0$. After few lines of computations not mentioned here, one also obtains

$$
\frac{\partial L}{\partial \tau}(\tau)=-\frac{q \delta_{m e} L(\tau)}{\left(\delta_{e}+L(\tau)\right)\left(1+\left(\frac{E^{*}(\tau)}{\beta}\right)^{q}\right)}\left[\delta_{e}-\frac{A K_{c}}{\beta}\left(\frac{E^{*}(\tau)}{\beta}\right)^{q-1} e^{-\tau \delta_{m e}}\right] .
$$

This expression enables us to study the variation of $L$ with respect to $\tau$. From

$$
\frac{A K_{c}}{\delta_{e}} \leq E^{*}(\tau=0) \leq \frac{A\left(K_{c}+\alpha\right)}{\delta_{e}},
$$

we establish that if model parameters are such that Condition (14) holds then $\tau \mapsto L(\tau)$ admits a unique maximum at

$$
\tilde{\tau}=\frac{1}{\delta_{m e}}\left[\frac{q-1}{2 q} \ln \left(1+\frac{\alpha}{K_{c}}\right)+\ln \left(\frac{A K_{c}}{\beta \delta_{e}}\right)\right],
$$

and

$$
L(\tilde{\tau})=\frac{q \delta_{e} \alpha}{2 K_{c}\left(1+\sqrt{1+\frac{\alpha}{K_{c}}}\right)+\alpha} .
$$

Continuity and variations with respect to $\delta_{e}$. Remembering the fact $L$ is strictly positive for all $\delta_{e}>0$, one can establish that $\delta_{e} \mapsto E^{*}\left(\delta_{e}\right)$ is continuously differentiable on $(0,+\infty)$ and

$$
\frac{\partial E^{*}}{\partial \delta_{e}}\left(\delta_{e}\right)=-\frac{E^{*}\left(\delta_{e}\right)}{\delta_{e}+L\left(\delta_{e}\right)} .
$$

$\delta_{e} \mapsto E^{*}\left(\delta_{e}\right)$ is thus a strictly decreasing ranging from $+\infty$ (when $\delta_{e} \rightarrow 0$ ) to 0 . One also obtains

$$
\frac{\partial L}{\partial \delta_{e}}\left(\delta_{e}\right)=L\left(\delta_{e}\right) \frac{(q+1)\left(\frac{E^{*}\left(\delta_{e}\right)}{\beta}\right)^{q}-(q-1)}{\left(\delta_{e}+L\left(\delta_{e}\right)\right)\left(1+\left(\frac{E^{*}\left(\delta_{e}\right)}{\beta}\right)^{q}\right)} .
$$

We establish after a few tedious computations that the function $\delta_{e} \mapsto L\left(\delta_{e}\right)$ admits a unique maximum

$$
L\left(\tilde{\delta}_{e}\right)=\frac{A \alpha e^{-\tau \delta_{m e}}}{4 \beta} \frac{(q-1)^{\frac{q-1}{q}}(q+1)^{\frac{q+1}{q}}}{q},
$$

with

$$
\tilde{\delta}_{e}=\frac{A e^{-\tau \delta_{m e}}}{\beta}\left(\frac{q+1}{q-1}\right)^{\frac{1}{q}}\left(K_{c}+\frac{3 \alpha}{5}\right)
$$


Continuity and variations with respect to $K_{c}$. As previously done, one can establish that the function $K_{c} \mapsto E^{*}\left(K_{c}\right)$ is continuously differentiable and

$$
\frac{\partial E^{*}}{\partial K_{c}}\left(K_{c}\right)=\frac{\delta_{e} E^{*}\left(K_{c}\right)}{\left(\delta_{e}+L\left(K_{c}\right)\right)\left(1+\left(\frac{E^{*}\left(K_{c}\right)}{\beta}\right)^{q}\right)} .
$$

The equilibrium $E^{*}$ is thus a strictly increasing function of $K_{c}$ and we have. One subsequently conclude, by composition, that $K_{c} \mapsto L\left(K_{c}\right)$ is continuously differentiable on $\mathbb{R}_{+}$, all other model parameters being fixed. With computations similar to the one performed by the reader in the latter paragraph, few lines of computations, not mentioned here, leads to

$$
\frac{\partial L}{\partial K_{c}}\left(K_{c}\right)=-\delta_{e} L\left(K_{c}\right) \frac{(q+1)\left(\frac{E^{*}\left(K_{c}\right)}{\beta}\right)^{q}-(q-1)}{\left(\delta_{e}+L\left(K_{c}\right)\right)\left(1+\left(\frac{E^{*}\left(K_{c}\right)}{\beta}\right)^{q}\right)\left(K_{c}+\frac{\alpha}{1+\left(\frac{E^{*}\left(K_{c}\right)}{\beta}\right)^{q}}\right)} .
$$

From this expression, one can study the variation of $L$ with respect to $K_{c}$. Using Eq. (9), one sees that

$$
E^{*}\left(K_{c}=0\right) \leq \frac{A \alpha \mathrm{e}^{-\tau \delta_{m e}}}{\delta_{e}}
$$

and easily establish that if the sufficient condition (13) holds, then $K_{c} \mapsto L\left(K_{c}\right)$ admits a maximum at

$$
\tilde{K}_{c}=\frac{\beta}{A} \tau \delta_{\mathrm{me}}\left(\frac{q-1}{q+1}\right)-\frac{\alpha(q+1)}{2 q}
$$

given by

$$
L\left(\tilde{K}_{c}\right)=A \alpha \mathrm{e}^{-\tau \delta_{m e}} \frac{(q+1)^{2}}{4 \beta q}\left(\frac{q-1}{q+1}\right)^{\frac{q-1}{q}} .
$$

Continuity and variations with respect to $\delta_{m e}$. Similarly to the previous paragraphs, we have that $\delta_{m e} \mapsto E^{*}\left(\delta_{m e}\right)$ is continuously differentiable and

$$
\frac{\partial E^{*}}{\partial \delta_{m e}}\left(\delta_{m e}\right)=-\frac{\tau \delta_{e} E^{*}\left(\delta_{m e}\right)}{\delta_{e}+L\left(\delta_{m e}\right)}<0 .
$$

Then, $\delta_{m e} \mapsto E^{*}\left(\delta_{m e}\right)$ is a strictly decreasing with $\lim _{\delta_{m e} \rightarrow \infty} E^{*}\left(\delta_{m e}\right)=0$. Similarly, one also obtains

$$
\begin{aligned}
\frac{\partial L}{\partial \delta_{m e}}\left(\delta_{m e}\right)=- & \frac{q \delta_{m e} L\left(\delta_{m e}\right)}{\left(\delta_{e}+L\left(\delta_{m e}\right)\right)\left(1+\left(\frac{E^{*}\left(\delta_{m e}\right)}{\beta}\right)^{q}\right)} \\
& \times\left(\delta_{e}-\frac{A K_{c}}{\beta}\left(\frac{E^{*}\left(\delta_{m e}\right)}{\beta}\right)^{q-1} e^{-\tau \delta_{m e}}\right) .
\end{aligned}
$$

With the same arguments previously mentioned, if Condition (14) is satisfied then $\delta_{m e} \mapsto L\left(\delta_{m e}\right)$ admits a maximum

$$
L\left(\tilde{\delta}_{m e}\right)=\frac{q \delta_{e} \alpha}{2 K_{c}\left(1+\sqrt{1+\frac{\alpha}{K_{c}}}\right)+\alpha},
$$

at

$$
\tilde{\delta}_{m e}=\frac{1}{\tau}\left[\frac{q-1}{2 q} \ln \left(1+\frac{\alpha}{K_{c}}\right)+\ln \left(\frac{A K_{c}}{\beta \delta_{e}}\right)\right] .
$$




\section{Appendix 3 : proof of Proposition 4.6}

Let $\psi$ be one of the parameter among $\delta_{e}, K_{c}, \delta_{m e}$ or $\tau$. First, let us remark that 0 is a root of Eq. (12) if and only if $\delta_{e}=-L(\psi)$. Yet, we know that $L(\psi)>0$. Consequently 0 cannot be a root Eq. (12). Moreover, considering the complex conjugate of Eq. (12), we obtain that if $\lambda \in i \mathbb{R}$ is a root of Eq. (12) then $-\lambda$ is also a root of this equation. From this, we know that if pure imaginary roots of Eq. (12) exist, then they will be given by $\lambda= \pm \mathrm{i} \omega(\psi), \omega(\psi)>0$.

Let $\psi^{*} \in \mathcal{P}$ be a chosen parameter. Thanks to Proposition 4.1 and to Corollary 4.1, we know that if $\psi^{*} \notin \Pi$ then pure imaginary roots of Eq. (12) cannot exist. It is thus necessary that $\psi^{*} \in \Pi$.

We begin to show the first part of Proposition 4.6. From what we just mentioned above, we assume, without loss of generality, that $i \omega\left(\psi^{*}\right), \omega\left(\psi^{*}\right)>0$, is a root of Eq. (12). In such situation, separating the real from the imaginary part, we obtain that $\omega\left(\psi^{*}\right)$ must verify

$$
\left\{\begin{aligned}
\delta_{e} & =-L\left(\psi^{*}\right) \cos \left(\omega\left(\psi^{*}\right) \tau\right) \\
\omega\left(\psi^{*}\right) & =L\left(\psi^{*}\right) \sin \left(\omega\left(\psi^{*}\right) \tau\right)
\end{aligned}\right.
$$

Considering that $-1 \leq \cos (x) \leq 1$ and that $\delta_{e}>0$, we verify that $\psi^{*} \in \Pi$ is a necessary condition for the definition of System (24). The latter system also reads

$$
\left\{\begin{array}{l}
\cos \left(\omega\left(\psi^{*}\right) \tau\right)=-\frac{\delta_{e}}{L\left(\psi^{*}\right)}, \\
\sin \left(\omega\left(\psi^{*}\right) \tau\right)=\frac{\omega\left(\psi^{*}\right)}{L\left(\psi^{*}\right)},
\end{array}\right.
$$

and we obtain

Consequently

$$
1=\cos \left(\omega\left(\psi^{*}\right) \tau\right)^{2}+\sin \left(\omega\left(\psi^{*}\right) \tau\right)^{2}=\left(\frac{\omega\left(\psi^{*}\right)}{L\left(\psi^{*}\right)}\right)^{2}+\left(\frac{\delta_{e}}{L\left(\psi^{*}\right)}\right)^{2}
$$

$$
\omega\left(\psi^{*}\right)^{2}=L\left(\psi^{*}\right)^{2}-\delta_{e}^{2},
$$

and we get that $\omega\left(\psi^{*}\right)$ is expressed by Eq. (16).

Remark 4. It is necessary that $\psi^{*} \in \Pi$ otherwise $\omega\left(\psi^{*}\right)$ is not defined. This condition also guarantee, a posteriori, that the function $\psi \mapsto \omega(\psi)$ is well defined on the set $\Pi$.

Finally, from the signs involved in Eq. (24), it is necessary that

$$
\omega\left(\psi^{*}\right) \tau \in \cup_{k \in \mathbb{N}}\left(\frac{(4 k+1)}{2} \pi,(2 k+1) \pi\right),
$$

from which it follows that there exists $k \in \mathbb{N}$ such that $\psi^{*} \in \Pi$ verifies

$$
\omega\left(\psi^{*}\right) \tau=\arctan \left(-\frac{\omega\left(\psi^{*}\right)}{\delta_{e}}\right)+(2 k+1) \pi .
$$

We reformulate the latter condition using the function $z$ defined in Eq. (17) and obtain the first part of Proposition 4.6: if $\mathrm{i} \omega\left(\psi^{*}\right)$ for $\psi^{*} \in \Pi$ is a root of (12) then there exists $k \in \mathbb{N}$ such that $\psi^{*} \in \Pi$ is a zero of $z(\cdot, k)$.

Remark 5. For all $k \in \mathbb{N}, \psi \mapsto z(\psi, k)$ is well defined and continuously differentiable on $\Pi$.

The proof of this remark directly originates from the continuous differentiability of $\psi \mapsto L(\psi)$ and $\psi \mapsto \omega(\psi)$ on $\Pi$.

The reciprocal of Proposition 4.6 is simply established by separating real and imaginary parts and by doing similar computations to the one above. The proof of the end of this proposition is given by Beretta and Kuang (2002).

Acknowledgements We are very thankful to Michael C. Mackey for his highly valuable feedback, comments and spelling corrections. 


\section{References}

Adimy M, Crauste F (2007) Modelling and Asymptotic Stability of a Growth Factor-Dependent Stem Cells Dynamics Model with Distributed Delay. Discrete and Continuous Dynamical Systems - Series B 8(1):19-38, DOI 10.3934/dcdsb.2007.8.19, URL https://hal.archives-ouvertes.fr/ hal-00258392, publisher: American Institute of Mathematical Sciences

Adimy M, Crauste F (2012) Delay Differential Equations and Autonomous Oscillations in Hematopoietic Stem Cell Dynamics Modeling. Math Model Nat Phenom 7(6):1-22, DOI 10.1051/mmnp/ 20127601, URL https://doi.org/10.1051/mmnp/20127601

Adimy M, Crauste F, Ruan S (2005) Stability and Hopf bifurcation in a mathematical model of pluripotent stem cell dynamics. Nonlinear Analysis: Real World Applications 6(4):651 670, DOI https://doi.org/10.1016/j.nonrwa.2004.12.010, URL http://www.sciencedirect.com/ science/article/pii/S1468121804001208

Adimy M, Crauste F, Ruan S (2006) Modelling Hematopoiesis Mediated by Growth Factors With Applications to Periodic Hematological Diseases. Bulletin of Mathematical Biology 68(8):2321-2351, DOI 10.1007/s11538-006-9121-9, URL https://doi.org/10.1007/s11538-006-9121-9

Banks HT, Cole CE, Schlosser PM, Tran HT (2004) Modeling and optimal regulation of erythropoiesis subject to benzene intoxication. Mathematical Biosciences \& Engineering 1(1):15-48, DOI 10.3934/ mbe.2004.1.15, URL https://www .aimsciences.org/article/doi/10.3934/mbe. 2004.1.15

Bélair J, Mackey MC, Mahaffy JM (1995) Age-structured and two-delay models for erythropoiesis. Mathematical Biosciences 128(1):317-346, DOI 10.1016/0025-5564(94)00078-E, URL http://www . sciencedirect.com/science/article/pii/002555649400078E

Beretta E, Kuang Y (2002) Geometric stability switch criteria in Delay Differential systems with Delay Dependent Parameters. SIAM Journal on Mathematical Analysis 33:1144-1165

Beutler E, Lichtman M, Coller B, Kipps T, Seligsohn U (2001) Williams Hematology, sixth edn. No. vol. 487 in Williams Hematology, McGraw-Hill, URL https://books.google.fr/books?id= VoFNCAAACAAJ

Björkholm M, Holm G, Merk K (1982) Cyclic autoimmune hemolytic anemia as a presenting manifestation of splenic Hodgkin's disease. Cancer 49(8):1702-1704, DOI 10.1002/1097-0142(19820415)49:8〈1702::AID-CNCR2820490827>3.0.CO;2-I, URL https: //acs journals.onlinelibrary.wiley.com/doi/abs/10.1002/1097-0142\%2819820415\%2949\% 3A8\%3C1702\%3A\%3AAID-CNCR2820490827\%3E3.0. CO\%3B2-I

Boullu L, Pujo-Menjouet L, Bélair J (2019a) Stability analysis of an equation with two delays and application to the production of platelets. Discrete and Continuous Dynamical Systems - Series S pp 1-24, DOI 10.3934/dcdss.2020131, URL https://hal.inria.fr/hal-02109546, publisher: American Institute of Mathematical Sciences

Boullu L, Pujo-Menjouet L, Wu J (2019b) A Model for Megakaryopoiesis with State-Dependent Delay. SIAM Journal on Applied Mathematics 79(4):1218-1243, DOI 10.1137/18M1201020, URL https: //doi.org/10.1137/18M1201020

Burwell EL, Brickley BA, Finch CA (1953) Erythrocyte Life Span in Small Animals Comparison of Two Methods Employing Radioiron. American Journal of Physiology-Legacy Content 172(3):718-724, DOI 10.1152/ajplegacy.1953.172.3.718, URL https ://doi .org/10.1152/ajplegacy.1953.172.3. 718, publisher: American Physiological Society

Butina M (2020) 5 - Erythrocyte production and destruction. In: Rodak's Hematology, sixth edition edn, Elsevier, St. Louis (MO), pp 62 - 77, DOI 10.1016/B978-0-323-53045-3.00014-3, URL http: //www.sciencedirect.com/science/article/pii/B9780323530453000143 
Craig M, Humphries AR, Mackey MC (2016) A Mathematical Model of Granulopoiesis Incorporating the Negative Feedback Dynamics and Kinetics of G-CSF/Neutrophil Binding and Internalization. Bulletin of Mathematical Biology 78(12):2304-2357, DOI 10.1007/s11538-016-0179-8, URL https : //doi.org/10.1007/s11538-016-0179-8

Crauste F (2010) Stability and Hopf Bifurcation for a First-Order Delay Differential Equation with Distributed Delay. In: Atay FM (ed) Complex Time-Delay Systems: Theory and Applications, Springer Berlin Heidelberg, Berlin, Heidelberg, pp 263-296

Crauste F, Pujo-Menjouet L, Génieys S, Molina C, Gandrillon O (2008) Adding self-renewal in committed erythroid progenitors improves the biological relevance of a mathematical model of erythropoiesis. Journal of Theoretical Biology 250(2):322-338, DOI 10.1016/j.jtbi.2007.09.041, URL http://www.sciencedirect.com/science/article/pii/S0022519307004778

Dale DC, Hammond WP (1988) Cyclic neutropenia: A clinical review. Blood Reviews 2(3):178 185, DOI https://doi.org/10.1016/0268-960X(88)90023-9, URL http://www. sciencedirect.com/ science/article/pii/0268960X88900239

Dinant HJ, Maat CEMd (1978) Erythropoiesis and Mean Red-Cell Lifespan in Normal Subjects and in Patients with the Anaemia of Active Rheumatoid Arthritis. British Journal of Haematology 39(3):437-444, DOI 10.1111/j.1365-2141.1978.tb01114.x, URL https://onlinelibrary.wiley. com/doi/abs/10.1111/j.1365-2141.1978.tb01114.x

Doig K (2015) Erythrocyte Production and Destruction. In: Rodak's Hematology -Clinical Principles and Applications, 5th edn, Elsevier Health Sciences, pp 95-111

Dou Y, Kruse A, Kotanko P, Rosen H, Levin NW, Thijssen S (2012) Red blood cell life span and 'erythropoietin resistance'. Kidney International 81(12):1275-1276, DOI 10.1038/ki.2012.54, URL https://www.kidneyinternational-online.org/article/S0085-2538(15)55260-5/abstract

Erslev AJ (1997) Clinical erythrokinetics: a critical review. Blood Reviews 11(3):160-167, DOI 10.1016/S0268-960X(97)90011-4, URL http://www.sciencedirect.com/science/article/pii/ S0268960X97900114

Finch CA (1982) Erythropoiesis, erythropoietin, and iron. Blood 60(6):1241-1246, DOI 10. 1182/blood.V60.6.1241.1241, URL https://ashpublications.org/blood/article/60/6/1241/ 162987/Erythropoiesis-erythropoietin-and-iron, publisher: American Society of Hematology

Finch CA, Deubelbeiss K, Cook JD, Eschbach JW, Harker LA, Funk DD, Marsaglia G, Hillman RS, Slichter S, Adamson JW, Ganzoni A, Biblett ER (1970) Ferrokinetics in man. Medicine 49(1):17-53, DOI 10.1097/00005792-197001000-00002

Foley C, Mackey MC (2009) Dynamic hematological disease: a review. J Math Biol 58(1):285-322, DOI 10.1007/s00285-008-0165-3, URL https://doi.org/10.1007/s00285-008-0165-3

Fontenele LPS, Santucci R, Centrone R, Aranha MAF, Bellesso M, Dias DF (2015) Cyclic Thrombocytopenia: A Case Report. Blood 126(23):4661-4661, DOI 10.1182/blood.V126.23.4661.4661, URL https://doi.org/10.1182/blood.V126.23.4661.4661

Fuertinger DH, Kappel F, Thijssen S, Levin NW, Kotanko P (2013) A model of erythropoiesis in adults with sufficient iron availability. J Math Biol 66(6):1209-1240, DOI 10.1007/s00285-012-0530-0, URL https://doi.org/10.1007/s00285-012-0530-0

Gibson CM, Gurney CW, Gaston EO, Simmons EL (1984) Cyclic erythropoiesis in the S1/S1d mouse. Experimental hematology 12(5):343-348

Gibson CM, Gurney CW, Simmons EL, Gaston EO (1985) Further studies on cyclic erythropoiesis in mice. Experimental hematology 13(9):855-860 
Goodnough LT (2002) The role of iron in erythropoiesis in the absence and presence of erythropoietin therapy. Nephrol Dial Transplant 17(suppl_5):14-18, DOI 10.1093/ndt/17.suppl_5.14, URL https : //academic.oup.com/ndt/article/17/suppl_5/14/1893541, publisher: Oxford Academic

Gordon RR, Varadi S (1962) Congenital hypoplastic anaemia (pure red-cell anaemia) with periodic erythroblastopenia. The Lancet 279(7224):296-299, DOI 10.1016/S0140-6736(62)91245-X, URL http://www.sciencedirect.com/science/article/pii/S014067366291245X

Greer JP, Foerster J, Lukens JN, Rodgers GM, Paraskevas F, Glader B (2003) Wintrobe's Clinical Hematology, vol 1, 11th edn. Lippincott Williams \& Wilkins, Philadelphia

Grover A, Mancini E, Moore S, Mead AJ, Atkinson D, Rasmussen KD, O'Carroll D, Jacobsen SEW, Nerlov C (2014) Erythropoietin guides multipotent hematopoietic progenitor cells toward an erythroid fate. J Exp Med 211(2):181-188, DOI 10.1084/jem.20131189, URL https://rupress.org/ jem/article/211/2/181/41590/Erythropoietin-guides-multipotent-hematopoietic

Gurney C, Simmons E, Gaston E (1981) Cyclic erythropoiesis in W/Wv mice following a single small dose of 89Sr. Experimental hematology 9(2):118-122, URL http://europepmc.org/abstract/ $\mathrm{MED} / 7238647$

Haurie C, Dale DC, Mackey MC (1998) Cyclical Neutropenia and Other Periodic Hematological Disorders: A Review of Mechanisms and Mathematical Models. Blood 92(8):26292640, DOI 10.1182/blood.V92.8.2629, URL https://ashpublications.org/blood/article/92/ 8/2629/261414/Cyclical-Neutropenia-and-Other-Periodic, publisher: American Society of Hematology

Hayes ND (1950) Roots of the Transcendental Equation Associated with a Certain DifferenceDifferential Equation. Journal of the London Mathematical Society s1-25(3):226-232, DOI 10.1112/jlms/s1-25.3.226, URL https://londmathsoc.onlinelibrary.wiley.com/doi/abs/10. $1112 / j 1 \mathrm{~ms} / \mathrm{s} 1-25.3 .226$

Jandl JH (1987) Blood : Textbook of Hematology. Lippincott Williams and Wilkins

Koury MJ, Bondurant MC (1990) Erythropoietin retards DNA breakdown and prevents programmed death in erythroid progenitor cells. Science 248(4953):378-381, DOI 10.1126/science.2326648, URL https://science.sciencemag.org/content/248/4953/378, publisher: American Association for the Advancement of Science Section: Reports

Krzyzanski W, Perez-Ruixo JJ, Vermeulen A (2008) Basic pharmacodynamic models for agents that alter the lifespan distribution of natural cells. J Pharmacokinet Pharmacodyn 35(3):349, DOI 10.1007/s10928-008-9092-6, URL https://doi .org/10.1007/s10928-008-9092-6

Langlois GP, Craig M, Humphries AR, Mackey MC, Mahaffy JM, Bélair J, Moulin T, Sinclair SR, Wang L (2017) Normal and pathological dynamics of platelets in humans. Journal of Mathematical Biology 75(6):1411-1462, DOI 10.1007/s00285-017-1125-6, URL https://doi.org/10.1007/ s00285-017-1125-6

Lichtman M, Beutler E, Thomas J Kipps M, Williams W, Kaushansky K, Kipps T, Seligsohn U (2006) Williams Hematology, Seventh Edition, 7th edn. McGraw-Hill medical publishing division, McGraw-Hill, New-York

Lodish H, Flygare J, Chou S (2010) From stem cell to erythroblast: Regulation of red cell production at multiple levels by multiple hormones. IUBMB Life 62(7):492-496, DOI 10.1002/iub.322, URL https://iubmb.onlinelibrary.wiley.com/doi/abs/10.1002/iub.322 
Loeffler M, Pantel K, Wulff H, Wichmann HE (1989) A mathematical model of erythropoiesis in mice and rats Part 1: Structure of the model. Cell Proliferation 22(1):13-30, DOI 10. 1111/j.1365-2184.1989.tb00198.x, URL https://onlinelibrary.wiley.com/doi/abs/10.1111/ j.1365-2184.1989.tb00198.x

Ma J, Dou Y, Zhang H, Thijssen S, Williams S, Kuntsevich V, Ouellet G, Wong MMY, Persic V, Kruse A, Rosales L, Wang Y, Levin NW, Kotanko P (2017) Correlation between Inflammatory Biomarkers and Red Blood Cell Life Span in Chronic Hemodialysis Patients. BPU 43(1-3):200-205, DOI 10.1159/000452728, URL https://www.karger.com/Article/FullText/452728

Mackey MC (1978) Unified hypothesis for the origin of aplastic anemia and periodic hematopoiesis. Blood 51(5):941-956, URL http://europepmc.org/abstract/MED/638253

Mackey MC (1979) Periodic auto-immune hemolytic anemia: An induced dynamical disease. Bltn Mathcal Biology 41(6):829-834, DOI 10.1007/BF02462379, URL https://doi.org/10.1007/ BF02462379

Mackey MC (1997) 8. Mathematical models of hematopoietic cell replication and control. In: Case Studies in Mathematical Modeling-Ecology, Physiology and Cell Biology, New York, pp 151-182

Mackey MC (2001) Cell kinetic status of haematopoietic stem cells. Cell Proliferation 34(2):7183, DOI 10.1046/j.1365-2184.2001.00195.x, URL https://onlinelibrary.wiley.com/doi/abs/ $10.1046 / j .1365-2184.2001 .00195 . x$

Mackey MC, Glass L (1977) Oscillation and Chaos in Physiological Control Systems. Science 197(4300):287-289, DOI 10.1126/science.267326

Mahaffy JM, Bélair J, Mackey MC (1998) Hematopoietic Model with Moving Boundary Condition and State Dependent Delay: Applications in Erythropoiesis. Journal of Theoretical Biology 190(2):135146, DOI 10.1006/jtbi.1997.0537, URL http://www.sciencedirect.com/science/article/pii/ S0022519397905373

Meyer RJ, Hoffman R, Zanjani ED (1978) Autoimmune hemolytic anemia and periodic pure red cell aplasia in systemic lupus erythematosus. The American Journal of Medicine 65(2):342-345, DOI 10.1016/0002-9343(78)90829-X, URL http://www.sciencedirect.com/science/article/ pii/000293437890829X

Morley A (1969) Blood-Cell Cycles in Polycythæmia Vera. Australasian Annals of Medicine 18(2):124-126, DOI 10.1111/imj.1969.18.2.124, URL https://onlinelibrary.wiley.com/doi/ abs/10.1111/imj.1969.18.2.124

Morley A (1979) Cyclic hemopoiesis and feedback control. Blood cells 5(2):283-296, URL http: //europepmc.org/abstract/MED/299070

Morley A, Stohlman F (1969) Erythropoiesis in the Dog: The Periodic Nature of the Steady State. Science 165(3897):1025-1027, DOI 10.1126/science.165.3897.1025, URL https://science. sciencemag.org/content/165/3897/1025, publisher: American Association for the Advancement of Science

Morley A, Baikie A, Galton D (1967) Cyclic leucocytosis as evidence for retention of normal homoeostatic control in chronic granulocytic leukaemia. The Lancet 290(7530):1320-1323, DOI 10.1016/S0140-6736(67)90910-5, URL https : //doi.org/10.1016/S0140-6736(67) 90910-5, publisher: Elsevier

Myssina S, Huber SM, Birka C, Lang PA, Lang KS, Friedrich B, Risler T, Wieder T, Lang F (2003) Inhibition of Erythrocyte Cation Channels by Erythropoietin. JASN 14(11):2750-2757, DOI 10. 1097/01.ASN.0000093253.42641.C1, URL https://jasn.asnjournals.org/content/14/11/2750 
Orr JS, Kirk J, Gray KG, Anderson JR (1968) A Study of the Interdependence of Red Cell and Bone Marrow Stem Cell Populations. British Journal of Haematology 15(1):23-34, DOI 10.1111/j.1365-2141.1968.tb01509.x, URL http://onlinelibrary.wiley.com/doi/abs/10.1111/ j.1365-2141.1968.tb01509.x

Pujo-Menjouet L (2016) Blood Cell Dynamics: Half of a Century of Modelling. Math Model Nat Phenom 11(1):92-115, DOI https://doi.org/10.1051/mmnp/201611106

Pujo-Menjouet L, Bernard S, Mackey MC (2005) Long Period Oscillations in a G0 Model of Hematopoietic Stem Cells. SIAM Journal on Applied Dynamical Systems 4(2):312-332, DOI https://doi.org/10.1137/030600473

Ramakrishnan R, Cheung WK, Wacholtz MC, Minton N, Jusko WJ (2004) Pharmacokinetic and Pharmacodynamic Modeling of Recombinant Human Erythropoietin After Single and Multiple Doses in Healthy Volunteers. The Journal of Clinical Pharmacology 44(9):991-1002, DOI 10.1177/0091270004268411, URL http://accp1.onlinelibrary.wiley.com/doi/abs/10.1177/ 0091270004268411

Ranløv P, Videbæk A (1963) Cyclic Haemolytic Anaemia Synchronous with Pel-Ebstein Fever in a Case of Hodgkin's Disease. Acta Medica Scandinavica 174(5):583-588, DOI 10. 1111/j.0954-6820.1963.tb07957.x, URL https://onlinelibrary.wiley.com/doi/abs/10.1111/ j.0954-6820.1963.tb07957.x

Reimann HA (1971) Haemocytic periodicity and periodic disorders: Periodic neutropenia, thrombocytopenia, lymphocytosis and anaemia. Postgrad Med J 47(549):504-510, URL https://www.ncbi . nlm.nih.gov/pmc/articles/PMC2467202/

Rogg S, Fuertinger DH, Volkwein S, Kappel F, Kotanko P (2019) Optimal EPO dosing in hemodialysis patients using a non-linear model predictive control approach. J Math Biol DOI 10.1007/ s00285-019-01429-1, URL https://doi.org/10.1007/s00285-019-01429-1

Sato Y, Mizuguchi T, Shigenaga S, Yoshikawa E, Chujo K, Minakuchi J, Kawashima S (2012) Shortened Red Blood Cell Lifespan Is Related to the Dose of Erythropoiesis-Stimulating Agents Requirement in Patients on Hemodialysis. Therapeutic Apheresis and Dialysis 16(6):522-528, DOI 10.1111/j.1744-9987.2012.01089.x, URL https://onlinelibrary.wiley.com/doi/abs/10.1111/ j.1744-9987.2012.01089.x

Shampine LF, Thompson S (2001) Solving DDEs in Matlab. Applied Numerical Mathematics 37(4):441-458, DOI 10.1016/S0168-9274(00)00055-6, URL http://www.sciencedirect.com/ science/article/pii/S0168927400000556

Singh R, Grinenko T, Ramasz B, Franke K, Lesche M, Dahl A, Gassmann M, Chavakis T, Henry I, Wielockx B (2018) Hematopoietic Stem Cells but Not Multipotent Progenitors Drive Erythropoiesis during Chronic Erythroid Stress in EPO Transgenic Mice. Stem Cell Reports 10(6):1908-1919, DOI 10.1016/j.stemcr.2018.04.012, URL http://www.sciencedirect.com/ science/article/pii/S2213671118301802

Woo S, Krzyzanski W, Jusko WJ (2006) Pharmacokinetic and Pharmacodynamic Modeling of Recombinant Human Erythropoietin after Intravenous and Subcutaneous Administration in Rats. J Pharmacol Exp Ther 319(3):1297-1306, DOI 10.1124/jpet.106.111377, URL http://jpet. aspetjournals.org/content/319/3/1297

Wu H, Klingmüller U, Besmer P, Lodish HF (1995) Interaction of the erythropoietin and stem-cellfactor receptors. Nature 377(6546):242-246, DOI 10.1038/377242a0, URL https://www. nature. com/articles/377242a0 